\title{
A Deep Neural Network for Detecting Coronavirus Disease Using Chest X-Ray Images
}

\author{
Rajeev Kumar Gupta, Pandit Deendayal Energy University, Gandhinagar, India \\ Nilesh Kunhare, Amity University, India \\ Rajesh Kumar Pateriya, Maulana Azad National Institute Technology, India \\ Nikhlesh Pathik, Sagar Institute of Science and Technology, India \\ iD https://orcid.org/0000-0002-3303-8684
}

\begin{abstract}
COVID-19 is one of the leading causes of death worldwide in the year 2020 and was declared a pandemic by the World Health Organization (WHO). This virus affects all countries across the world and 5 lakh people died as of June 2020 due to COVID-19. Due to the highly contagious nature, early detection of this virus plays a vital role to break the covid chain. Recent studies done by China say that chest CT and X-ray image may be used as a preliminary test for covid detection. Deep learning-based CNN model can detect coronavirus automatically from the chest $\mathrm{X}$-ray images. This paper proposed a transfer learning-based approach to detect covid disease. Due to the low number of covid chest images, the authors are using a pre-trained model to classify x-ray images into covid and normal classes. This paper presents the comparative study of various pre-trained models like VGGNet-19, ResNet50, and Inception_ResNet_V2. Experiment results show that Inception_ResNet_V2 gives better results than VGGNet and ResNet models with training and test accuracy of 99.26 and 94, respectively.
\end{abstract}

\section{KEYWORDS}

CNN, COVID-19, Inception_ResNet_V2 and MobileNetV_2, Transfer Learning, VGG19

\section{INTRODUCTION}

An outbreak of a new family of the virus in December 2019, SARS- COV-2 (Severe Acute Respiratory Syndrome), has emerged as the leading cause of humans and animal mortality. The impact on public health and the global economy has been devastating. The complicated structure of the virus and zoonotic nature, it is very difficult to diagnosis at early stage. It has claimed more than 10 million lives, including 5 lakh deaths and 4.92 million recovered worldwide (till June 2020), thus compelled WHO to declare COVID - 19 as a pandemic in February 2020 (Ozturka T. et al., 2020). Table 1 depicts the top ten coronavirus affected countries in the world.

Figure 1 shows the distribution of Covid cases countrywide. COVID- 19 or COV family is exceptionally contagious and transmitted by droplets (Zu Z. Y et al., 2020; Khan A. I et al., 2020). If a person encounters an infected person while sneezing or coughing or being exposed to the contaminated surfaces. If infected, the person shows no evident symptoms in the early stages but gradually develops few symptoms like high fever, dry cough, sore throat, mild Pneumonia, but in later stages, the person

DOI: 10.4018/IJHISI.20220401.oa1

This article published as an Open Access article distributed under the terms of the Creative Commons Attribution License (http://creativecommons.org/licenses/by/4.0/) which permits unrestricted use, distribution, and production in any medium, provided the author of the original work and original publication source are properly credited. 
International Journal of Healthcare Information Systems and Informatics Volume $17 \cdot$ Issue 2

Table 1. Distribution of Covid-19 Cases (Top 10 Countries) (as on 27 June 2020)

\begin{tabular}{|c|l|c|c|c|}
\hline$\#$ & Country & Total Cases & Total Deaths & Total Recovered \\
\hline & World & $9,987,565$ & 498,733 & $5,410,132$ \\
\hline 1 & USA & $2,573,732$ & 127,845 & $1,070,367$ \\
\hline 2 & Brazil & $1,280,335$ & 56,121 & 697,526 \\
\hline 3 & Russia & 627,646 & 8,969 & 393,352 \\
\hline 4 & India & 528,136 & 16,096 & 309,829 \\
\hline 5 & UK & 310,250 & 43,514 & N/A \\
\hline 6 & Spain & 294,985 & 28,338 & N/A \\
\hline 7 & Peru & 272,364 & 8,939 & 159,806 \\
\hline 8 & Chile & 267,766 & 5,347 & 188,584 \\
\hline 9 & Italy & 240,136 & 34,716 & 180,661 \\
\hline 10 & Iran & 220,180 & 10,364 & \\
\hline
\end{tabular}

Figure 1. Countries Covid Cases Distribution

Ground glass opacities +/-consolidation

Pure consolidation

Multiple lesions

Bilateral involvement

Posterior part / lower lobe predilection

Peripheral / subpleural distribution

Crazy-paving pattern

Air bronchogram

Reversed halo sign on high-resolution CT

Pleural effusion

Cavitation, calcification, lymphadenopathy

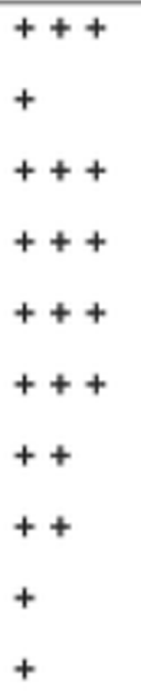

Absent 
suffers from the complete disappearance of the sense of smell and taste and severe form of respiratory complications accompanied by multi-organ failures leading to death.

The present scenario is facing with insufficiency of clinical diagnosis resources. Limited test kits \& ventilators are available and vaccines yet to be designed, which adds to the global situation's criticality. Thus, it encourages researchers and academicians to develop quick, convenient, accurate and reliable solutions to detect COVID-19.

The extended incubation period of the disease and the time taken to confirm the test outcomes may result in the diseases' spread. Nowadays, Artificial Intelligence (AI) and Machine Learning are heavily used in the medical domain. AI technology is mature enough and can be used to detect COVID-19 positive and negative cases. Asif Asnaoui K. E. \& Chawki Y. (2020) believe that AI-based techniques can be applied on radiologists images like X-rays, CT images may promptly deliver a promising and comprehensive detection of COVID-19.

RT-PCR (Real-time- Polymerase Chain Reaction) used as a standard solution to detect COVID-19 from radio logistic images (Khan A. I et al., 2020). It proved to be useful but not the best method because of low RT-PCR sensitivity (approximately 60\%- 70\%) (Ozturka T. et al., 2020), high falsenegative rate (Zu Z. Y et al., 2020), expensive technique (Khan A. I et al., 2020), and unavailability of the required assay for diagnosis of the disease ((Zu Z. Y et al., 2020). Due to the limited availability, high cost and time taking process of RT-PCR test, researchers shifted their interest to explore AI-based solutions to diagnose Covid using X-rays and CT scan images. Although chest X-ray is not a standard way to find Covid, it is used as significant evidence for Covid detection in Hubei. Zu Z. Y et al., 2020 says that chest X-ray images can be used for the early detection of Covid. This approach opened the doors for qualitative and quantitive analysis of COVID 19 cases and minimized RT-PCR limitations. Table 2 shows the different features of chest X-ray images that can be useful for Covid detection.

Table 2. Corona Virus Features in Chest CT Image (Zu Z. Y et al., 2020)

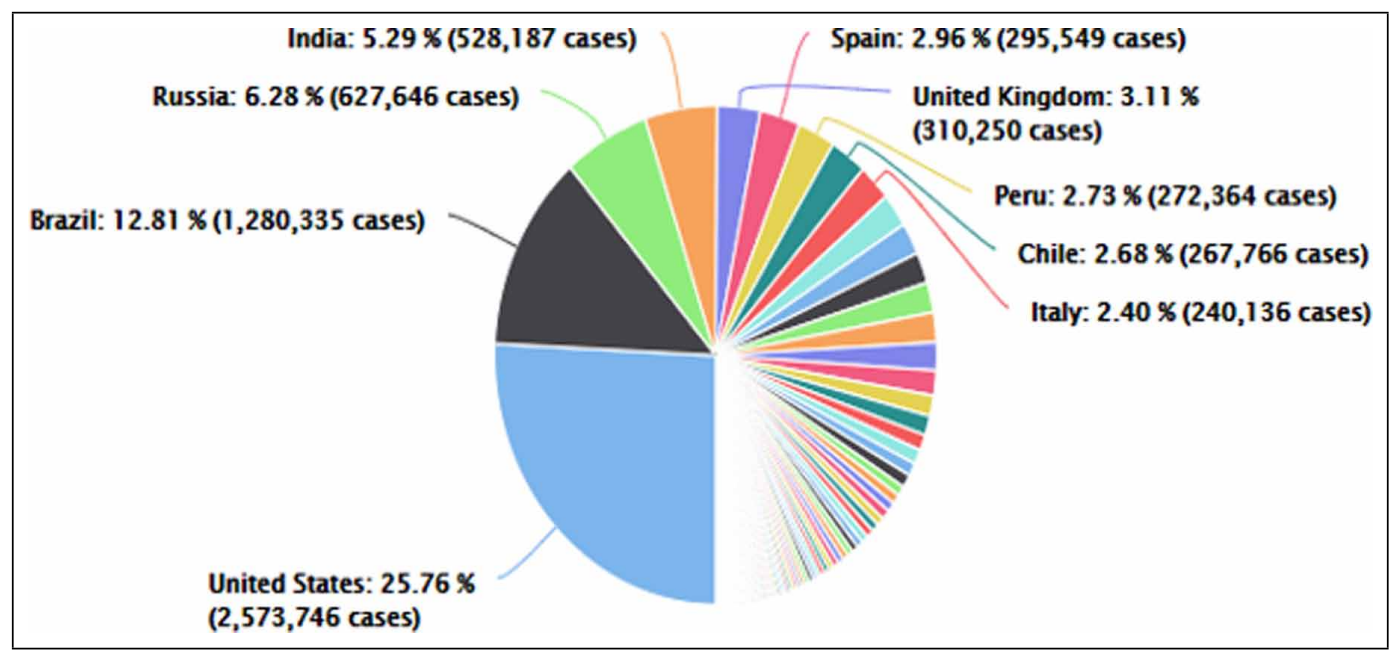

A recent study says that Covid patient in the early stage (mid Covid) can be recovered without the ICU and ventilator support. These medical facilities are required mainly for the covid severe patient. Table 3 shows the symptoms of mid-Covid and severe Covid patients.

These AI-based solutions can be used as a pre-milling test. If an individual has been classified as a Covid patient, more clinical trials can be performed to confirm a Covid patient. If the individual is 
Table 3. Covid Patient Symptoms (Asnaoui K. E. \& Chawki Y., 2020)

\begin{tabular}{|l|l|l|l|}
\hline \multicolumn{1}{|c|}{ Diseases } & $\begin{array}{l}\text { Respiratory } \\
\text { symptoms }\end{array}$ & $\begin{array}{l}\text { Constitutional } \\
\text { symptoms }\end{array}$ & CT imaging findings \\
\hline Mid Covid-19 & Cough or not, Sore Throat & Fever & $\begin{array}{l}\text { Multiple patchy GGOs with } \\
\text { subpleural distribution }\end{array}$ \\
\hline Severe Covid-19 & $\begin{array}{l}\text { Breathless, Respiratory } \\
\text { Failure }\end{array}$ & $\begin{array}{l}\text { Fever, Confusion, Muscle } \\
\text { Ache, Headache }\end{array}$ & $\begin{array}{l}\text { Diffuse heterogeneous } \\
\text { consolidation with GGO }\end{array}$ \\
\hline
\end{tabular}

identified as a Covid patient at an early stage, we can take safety measurements like home insulation to break the covid chain.

In this work, an AI based solution was designed to detect whether a person is a Covid patient or not. Due to the availability of fewer Covid X-ray images, we use a pre-trained model and customize it to make it appropriate for Covid detection. Since pre-trained model have number of layers which increase the chance of overfitting. To avoid overfitting, we have implemented image augmentation and regularization techniques. The main achievement of this work is that our model has very less false positive rate. The rest of the paper is structured as follows, Section 2 highlights on the relevant work and literature research. Section 3 explains the proposed model, Section 4 reveals the performance assessment of the proposed model and Section 5 ends the work as a whole and sets out guidelines for future work.

\section{RELATED WORK}

Recently, many Machine Learning-based exciting contributions have been cited for the detection and identification of COVID-19. Most of the work done so far uses Deep Convolution Neural Network (CNN) models and pre-trained models such as ChexNet (Rajpurkar P et al., 2017), ChestNet (Wang W. \& Xia Y., 2018) ResNet-50, VBNet, etc. They claim the accuracy ranging from $87 \%$ to $95 \%$. ResNet-50 was suggested by Gozes O. et al. (2020) to distinguish patients with coronavirus and healthy individuals by generating a localization map of lung abnormalities.

Bhandary A. et al. (2020), suggested two models for the classification of lung abnormalities-a) MAN combined with SVM for the detection of pneumonia images from normal images with $97.27 \%$ accuracy. b) MAN with EFT to increase the efficiency of the classifier with an accuracy of $86.47 \%$.

Narin A et al. (2020) and Hemdan E. Det al. (2020) also proposed their models CNN architecture (accuracy-98\%) and COVIDX-Net (accuracy- 89\% - 91\%) respectively to classify positive and negative COVID-19 patient by using pre-trained models. Model is trained on COVIDX-Net dataset. Farooq M. \& Hafeez A. (2020) proposed a CNN framework, named COVID_ResNes to identify Pneumonia (bacteria and virus) up to $83.5 \%$ accuracy.

Likewise, contributions were seen by Zhang J. et al. (2020) where they developed a simple deep neural network and achieved 86.7\% accuracy. Shan F. et al. (2020) have researched VB-Net Neural Network and HITL (Human in the Loop) iterative approaches for the detection of COVID-19. Other researchers have performed a comparative study of recent DCNN architectures for binary and multiclass X-ray classifications between normal bacteria and coronavirus.

Makris A. et al. (2020) introduced transfer learning-based solution for the Covid detection. This model is trained for three classes i.e., Normal, Covid and Pneumonia. This paper simply used some existing pre-trained model like VGG-16, VGG-19, ResNet152, MobileNet V2 etc. and claiming maximum accuracy of 95.8 by using VGG-19. In this paper, model is trained on a tiny dataset (approx. 70 images) and not used any augmentations technique to increase dataset size.

Asif S. et al. (2020), proposed Covid detection model using deep neural network and CNN. This paper used Inception_v3 pre-trained model to trained our model. This paper uses all X-ray images 
of Covid dataset which also contain the X-ray images of some other virus-like Acute Respiratory Distress Syndrome (ARDS), Severe Acute Respiratory Syndrome (SARS) etc. Besides, unbalance dataset is used to train a model which may lead to predicting the wrong classification.

Ilyas M.et al. (2020)], discuss various existing Deep Learning based approach used to detect Covid form X-ray images. This paper discussed various pre-trained model used for the Covid detection along with the dataset size which is used by the author for trained our network. Most of the network is trained on very less number of images even on 20 Covid images and augmentation techniques to increase dataset.

On the other hand, the authors Zu Z. Y et al. (2020) deeply analyzed the disease's epidemiological and epidemiological radiology. The authors Ozturka T. et al. (2020), proposed an end to end architecture without any feature extraction method with accuracy up to $98.08 \%$ and $87.02 \%$ for binary and multiclass tasks respectively. Saddam Hussain and Khan S. et al. (2020) proposed COVID ReNet and custom VCG-16 architecture that exploits edge and region-based classification in CNN up to $98.7 \%$ accuracy. Even to deal with radiological image irregularities and limited dataset on COVID-19 Abbas A. et al. (2020) adopted and validated DeTrac (Decompose, Transfer, Compose) class decomposition mechanism. Despite of minor Miscalibrations and tuning it gives accuracy up to $95.12 \%$.

\section{PROPOSED WORK}

The main aim of this research is to diagnose Covid-19 with X-ray images of the chest. For this reason, we use two publicly accessible datasets called chest X-ray \& CT dataset (Kermany et al., 2017) and Covid dataset (Cohen et al., 2020). A large number of images are needed for a trained deep neural network, but the Covid dataset contains only 738 chest X-ray images, so this study uses a pre-trained model called VGG-19, RestNet and InceptionNet to diagnose Covid. After removing the upper layers from the pre-trained model, new classification layers are added, which classify the chest X-ray images into two classes i.e. Covid and Normal, where the Covid class represents the patient and the Normal class represents a healthy individual. Figure 2 displays the block diagram of the suggested technique. The proposed approach involved the following phases.

a) Dataset Preparation

b) Pre-processing

c) Extract Pre-Trained Model Customization

d) Training Model

e) Performance Evaluation Metrics

Figure 2. Block Diagram of the Proposed Model

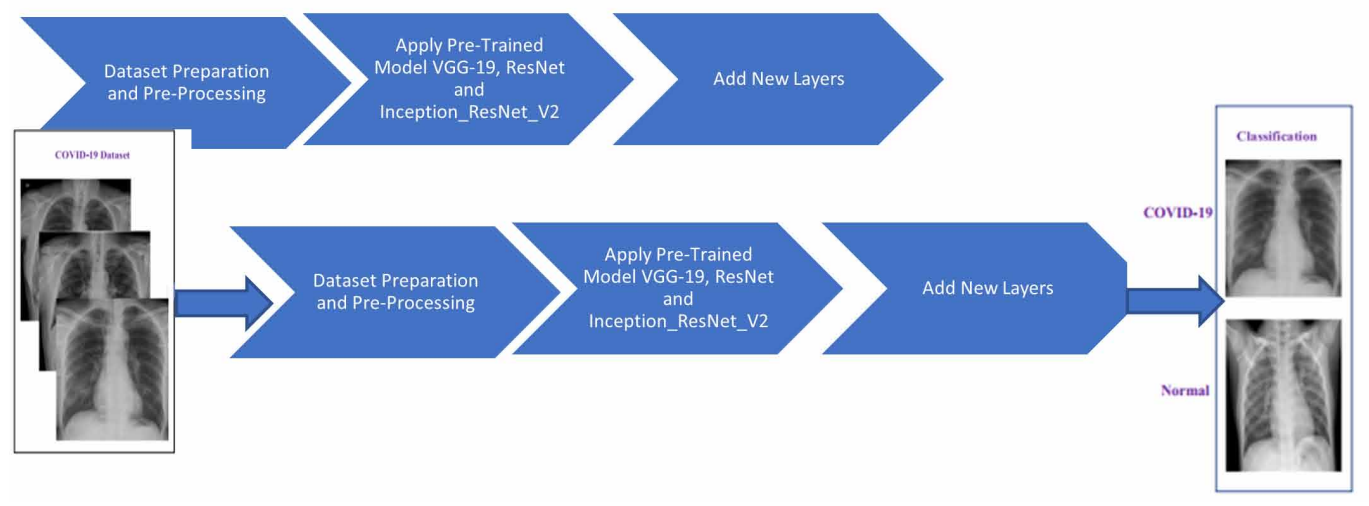




\subsection{Dataset Preparation}

The accuracy of the model depends mainly on the data collection. In this work, we use two publicly available datasets called chest X-ray \& CT (Kermany et al., 2017) and Covid19 Chest X-ray (Cohen et al., 2020). Chest X-ray \& CT dataset consists of 5856 X-ray images, which are divided into three parts: train, test and validation. This dataset is classified into two classes named Pneumonia class (4273 images) and Normal class (1583 images) (1583 images). Covid19 Chest X-ray dataset consists of 738 X-ray and CT images and updating regularly. These images are classified into four classes i.e., COVID-19, MERS, SARS, and ARDS. As the Covid dataset is updating regularly so proposed model can also be trained for the new dataset.

To prepare the dataset for our analysis, we mixed both datasets. Since we are only interested in classifying whether the individual is Covid or Normal, we have selected only Covid images with posteroanterior (PA) view from the Covid dataset. After extracting only Covid images, we get 206 Covid images with the PA view. Since the number of images in the chest X-ray dataset is 5856, if 206 Covid images are combined with 5856 chest X-ray images, our model is likely to overfit and favor the Normal class. To prevent over-fitting, we randomly picked 206 Normal images from the chest X-ray dataset. Hence, our final dataset includes 412 X-ray images (i.e., Covid: 206, Normal: 206) (i.e., Covid: 206, Normal: 206). We do not consider Pneumonia, MERS, SARS, and ARDS X-ray images during dataset preparation because our main objective of this study is to detect whether or not a person is suffering from Covid.

This study aims to minimize the false positive (i.e. the true Covid patient classified as a Normal person) because if the Covid patient classified as Normal, it would be more dangerous to society than vice versa. As our model is trained only for Covid and Normal images. So there is a high chance that X-ray image will be classified as Covid class because Pneumonia, MERS, SARS, and ARDS n $\mathrm{X}$-ray images are different from normal X-ray images.

Since the proposed method is used as a preliminary test so if the input image is diagnoses as a Covid class, further tests can be taken in order to confirm whether the person is suffering from Covid, Pneumonia, MERS, SARS, and ARDS. The proposed model will minimize the chances of classifying a patient as a healthy person. Pseudo code for dataset creation is given below

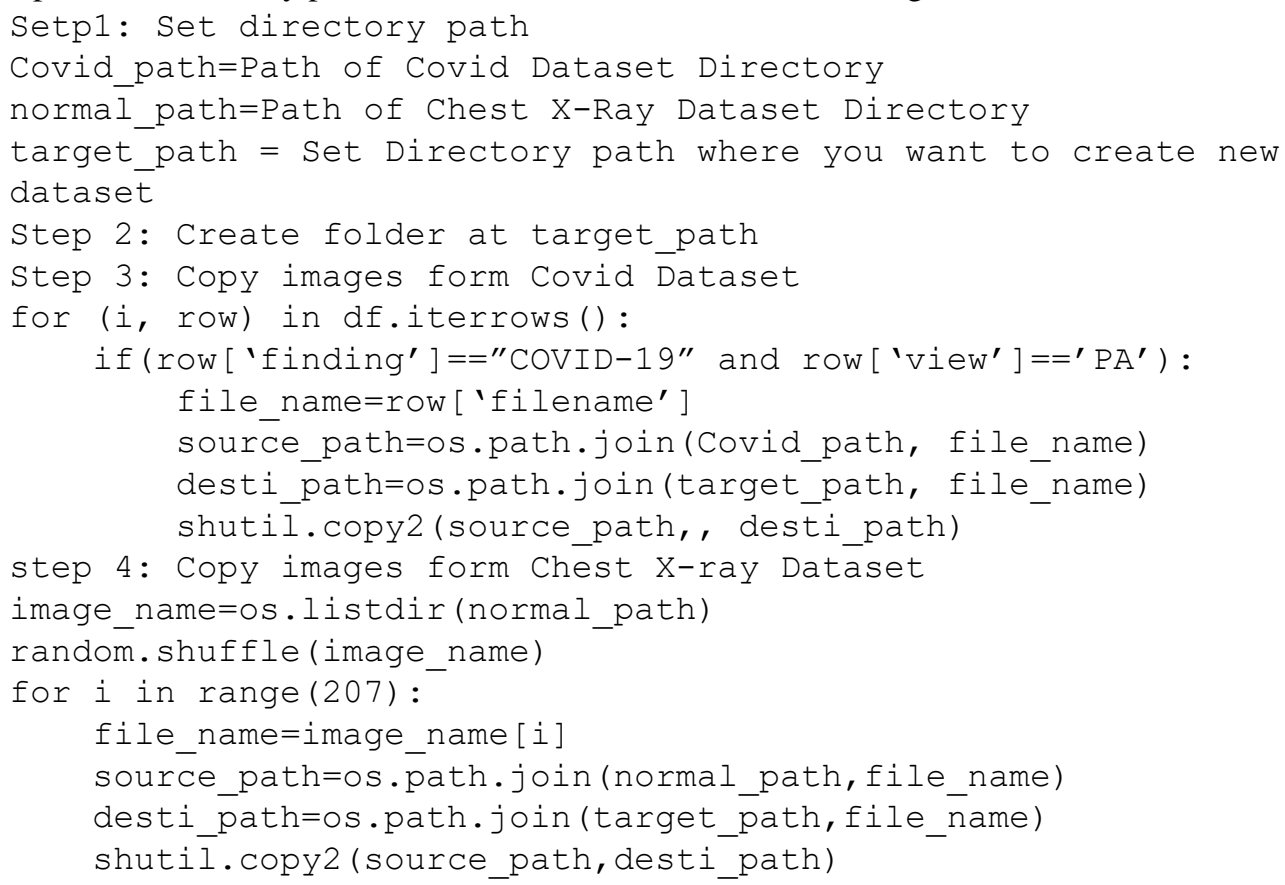


Table 4. Dataset Description before Augmentation

\begin{tabular}{|l|l|l|l|l|l|}
\hline & $\begin{array}{c}\text { No. of Covid } \\
\text { Images }\end{array}$ & $\begin{array}{c}\text { No. of Normal } \\
\text { Images }\end{array}$ & $\begin{array}{c}\text { No. of Pneumonia } \\
\text { Images }\end{array}$ & $\begin{array}{c}\text { No. of ARDS } \\
\text { Images }\end{array}$ & $\begin{array}{c}\text { No. of SARS } \\
\text { Images }\end{array}$ \\
\hline Total Images & 206 & 206 & 3 & 3 & 3 \\
\hline Train & 186 & 186 & 0 & 0 & 0 \\
\hline Validation & 20 & 20 & 0 & 0 & 0 \\
\hline Test & 3 & 3 & 3 & 3 & 3 \\
\hline
\end{tabular}

\subsection{Data Pre-Processing}

The size of the dataset plays a vital role in the learning process. Typically, the performance of the model depends on the size and accuracy of the dataset and increases with dataset size. The dataset divided into three parts i.e., train, validation and test. Train dataset consists of 186 Covid patient images and 186 images of a normal person. In contrast, the validation dataset consists of 20 Covid images and 20 Normal images. The main aim of this study is to tell that the person is not healthy (not Normal). So if the person is suffering from Pneumonia, Acute Respiratory Distress Syndrome (ARDS) and Severe Acute Respiratory Syndrome (SARS) it must be classified as a Covid patient because the symptom of these diseases is very similar. Because of this reason, we are not training our model for Pneumonia, ARDS and SARS but at the time of testing three images from each class (Pneumonia, ARDS, SARS, Covid and Normal) are pass to the model to measure the performance of the developed model. Table 4 depicts the dataset description before augmentation.

The image augmentation techniques are used to increase the size of the dataset. In the image augmentation, we are not rotating the image because it is hard to correctly identified the actual class from the rotated image, even for a human. Moreover, to improve the appearance of X-ray images, histogram equalization technique is used. Table 5 depicts the dataset description after augmentation.

Table 5. Dataset Description After Augmentation

\begin{tabular}{|l|l|l|l|l|l|}
\hline & $\begin{array}{c}\text { No. of Covid } \\
\text { Images }\end{array}$ & $\begin{array}{c}\text { No. of Normal } \\
\text { Images }\end{array}$ & $\begin{array}{c}\text { No. of Pneumonia } \\
\text { Images }\end{array}$ & $\begin{array}{c}\text { No. of ARDS } \\
\text { Images }\end{array}$ & $\begin{array}{c}\text { No. of SARS } \\
\text { Images }\end{array}$ \\
\hline Total Images & 2060 & 2059 & 3 & 3 & 3 \\
\hline Train & 1860 & 1859 & 0 & 0 & 0 \\
\hline Validation & 200 & 200 & 0 & 0 & 3 \\
\hline Test & 3 & 3 & 3 & 3 & 3 \\
\hline
\end{tabular}

Figure 3 depicts the X-ray images of a Covid patient and a Normal person. It is observed from figure 3 that Normal X-ray images have both clear lungs with no zones of abnormal opacification. In contrast, Covid X-ray image shows a diffuse interstitial pattern in both lungs.

\subsection{Pre-trained Model Customization}

As a huge dataset is required to train deep neural network model. Due to availably of less number of Covid images, we are using pre-trained models and customized these models to diagnose Covid or Normal patients. The purpose of using a pre-trained model is to achieve higher accuracy with a small dataset. In this work, three pre-trained models are trained to classify X-ray images into Covid-19 or Normal images. These pre-trained models are 
Figure 3. Covid and Normal X-Ray Images
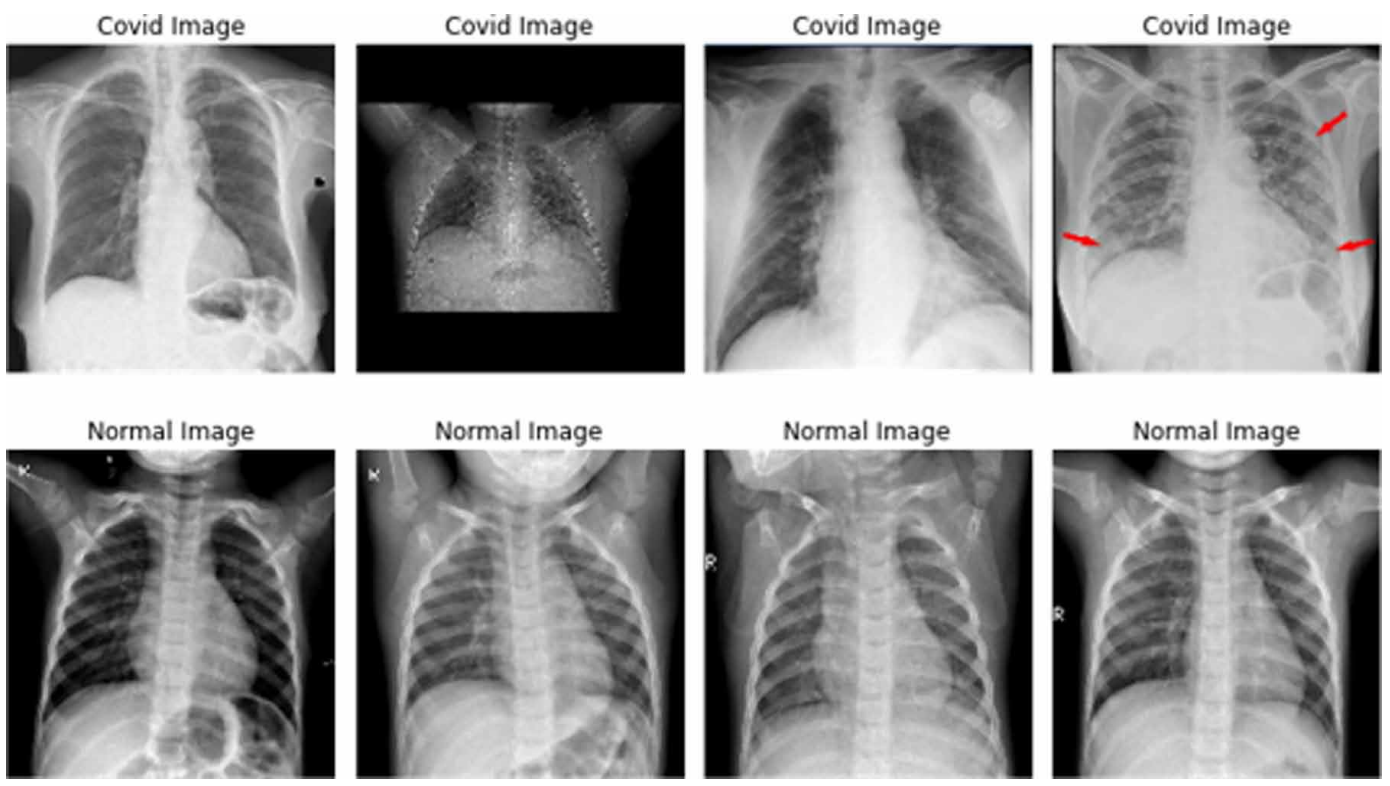
a) VGGNet-19
b) ResNet
c) Inception_resnet_V2

These all models are trained for ImageNet Large Scale Visual Recognition Challenge (ILSVRC) dataset (Simonyan K. \& Zisserman A., 2015). This dataset consists of 1.2 million training images, 100,000 test images and 50,000 images for validation and has 1000 different classes. All three models consist of several Convolution and Pooling layers where convolution layers are used to detect features and the pooling layer is responsible for minimizing the input image's size, which reduces the overfitting. If $\mathrm{I}(n \times m)$ is an input image, $\mathrm{k}$ is a kernel of size $(\mathrm{a}, \mathrm{b})$ then two-dimension convolution operation is given by

$$
S_{i j}=(I * K)_{i j}=\sum_{a=0}^{n-1} \sum_{b=0}^{m-1} I_{i+a, j+b} * K_{a, b}
$$

Where $S_{i j}$ represents the $\mathrm{i}^{\text {th }}$ and $\mathrm{j}^{\text {th }}$ pixel value of an image.

The hidden layer uses three types of activation functions named ReLu, LeakyReLu and Softmax. The mathematical equation for pooling operation and activation function is given in table 6 . Where out $_{i}$ represent the output of $\mathrm{i}^{\text {th }}$ pooling layer, $Z_{i}$ represent pre activation output of layer $\mathrm{i},(\mathrm{a}, \mathrm{b})$ is the size of filter.

To fine-tune our model to classify X-ray images into two classes (i.e., Covid and Normal), we removed top layers from all pre-trained models and added new layers to the model. First, add Global Average Pooling Layer (Lin M. et al., 2014) to minimize the number of trainable parameters. This layer will help to reduce overfitting. Global Average Pooling Layer is used just before the new classification layer. The pre-trained model's output is given to the Global Average Pooling Layer, which takes all features' average values. Global Average Pooling Layer reduces input dimension of 
Table 6. Mathematics Equation for Various CNN Operation

\begin{tabular}{|l|l|}
\hline \multicolumn{1}{|c|}{ Operation } & \multicolumn{1}{|c|}{ Equation } \\
\hline Max Pooling & out $_{i}=\max _{1<j<a \times b}\left(x_{j}\right)$ \\
\hline Avg. Pooling & out $_{i}=\operatorname{avg}_{1<j<a X b}\left(x_{j}\right)$ \\
\hline ReLu & $\operatorname{ReLU}\left(z_{i}\right)=\max \left(0, z_{i}\right)$ \\
\hline LeakyReLu & LeakyReLU $\left(z_{i}\right)=\max \left(0.01 z, z_{i}\right)$ \\
\hline Sigmoid & $\sigma\left(z_{i}\right)=\frac{1}{1+e^{-Z_{i}}}$ \\
\hline Softmax & $\operatorname{softmax}\left(z_{i}\right)=\frac{e^{Z_{i}}}{\sum_{j}^{Z_{j}}}$ \\
\hline
\end{tabular}

$h \times \mathrm{w} \times D$ to $1 \times 1 \times D$. This output is pass to three fully connected dense layers. Each dense layer having 1024 neurons and ReLU activation function. To minimize overfitting, 20\% of neuron s from each layer is dropout. Our model is binary, so the last layer of our model has only one neuron with a sigmoid activation function. Stochastic Gradient Descent optimizer with 0.01 learning rate and binary cross-entropy loss is set to compile the model. Pseudo code for the proposed model is given by

Step-1: Import pre- trained model

base_mdoel=tf.keras.applications.pre_trained_model _

name (weights=' imagenet', include_top=False)

Step-2: Design new model

$\mathrm{x}=\mathrm{base}$ moloel. output

Step-2.1: Add Global Average Pooling Layer

$\mathrm{x}=\mathrm{tf}$. keras.layers.GlobalAveragePooling2D () (x)

Step-2.2: Add nine dense layer with 1024 neuron and relu

activation function

$\mathrm{x}=\mathrm{tf}$. keras.layers. Dense (1024, activation='relu') (x)

Step-2.3: Apply regularization to avoid overfitting, drop 20\%

neuron from each layer

$\mathrm{x}=\mathrm{tf}$.keras.layers. Dropout (0.2) (x)

Step-2.4: Add one dense layer with 1 neuron and sigmoid

activation function

preds = tf.keras.layers.Dense(1, activation = 'sigmoid') $(\mathrm{x})$

vgg_model=tf.keras.models.Model(inputs = base_mdoel1.input,

outputs $=$ preds)

Step-3: Compile model with SGD optimizer and binary cross entropy

loss

$1=0.1, \mathrm{de}=.0000001, \mathrm{mo}=0.9$

opt=tf.keras.optimizers.SGD ( $1 r=1$, decay=d, momentum=mo, 


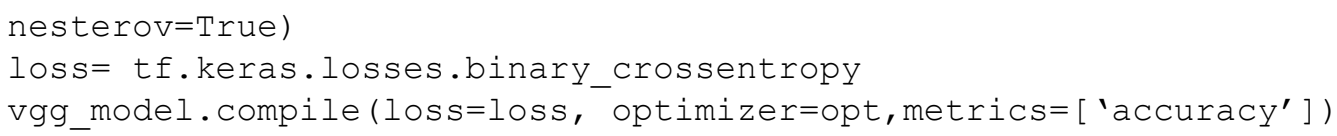

Each model has a certain number of layers with trained weight so in our proposed model, we only trained weight for the newly added layers. Description of all pre-trained model is given below.

\section{a) VGGNet- 19}

It is introduced by Simonyan K. \& Zisserman A. (2015). This model has 16 convolution layers with a filter size of 3x3, 5 max pooling layer and two fully connected layers. Each layer has 4096 neurons and one classification layer that used Softmax activation function and 1000 neurons to classify 1000 objects. Figure 4 shows the layered architecture of VGGNet-19.

After removing the top layer from the VGG-19 pre-trained model, new classification layers is added. The total number of layers in the new model is 32 (i.e., 22 layers from the pre-trained model and ten new layers). The proposed model is trained only for newly added layers.

\section{b) ResNet-50}

It is introduced by He K. et al. (2015). It is an intense neural network consisting of several building blocks instead of the traditional sequential network like VGGNet. This model has 175 convolution block and one fully connected dense layers with 1000 neurons and Softmax activation function. Figure 5 depicts the layered architecture of ResNet-50.

After the customization new model will have 185 layers (i.e., 175 layers from the pre-trained model and ten new layers). The proposed model will train only for newly added layers.

\section{c) InceptionResNetV2}

It is introduced by Szegedy C. et al. (2017). This model combines the features of the residual net (ResNet) and inception net. It is one of the deepest neural networks consisting of several inception blocks (1X1 convolution without activation). This model has 780 inception block and one fully connected dense layer with 1000 neurons and Softmax activation function. Figure 6 depicts the layered architecture of InceptionResNetV2.

After the model customization number of layers in the new model is 790 (i.e., 780 layers from the pre-trained model and ten new layers). The new model will train only for newly added layers.

\subsection{Training Model}

The next step after building a new model is to train this model. Because only the newly added layer needs to be trained, the proposed model takes less time to learn. Table 7 shows total layers, total parameters, number of trainable parameters and time required to train the model for ten epochs.

\subsection{Performance Evaluation Metrics}

Confusion matrix, Precision, Recall and F1 scores are used to test the efficiency of the model. The mathematical equation for all metrics is given below:

Confusion Matrix: It is one of the most common matrices for evaluating the efficiency of the classification model. It provides a full description of the real and expected outcome. It is a $n * n$ matrix that represents the number of correct and incorrect predictions. 
Figure 4. Layered Architecture of VGGNet-19

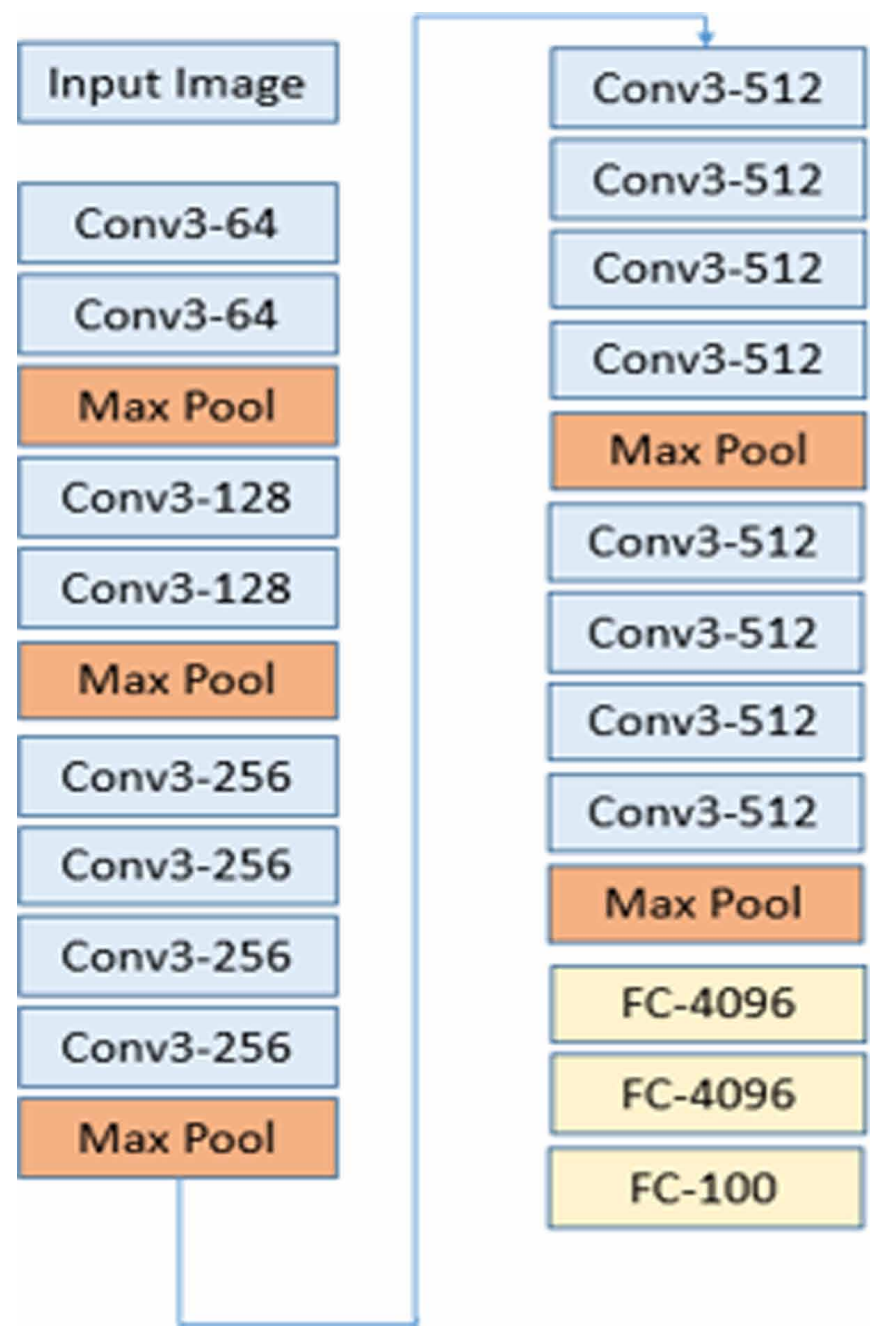

Accuracy: It represents the accuracy of the model. It is a ratio of total true prediction and overall prediction.

Accuracy $=\frac{T P+T N}{T P+T N+F P+F N}$

Precision: Precision shows the percentage of the relevant result. It is a ratio of true positive and total predictive positive.

Precision $=\frac{T P}{T P+F P}$ 
Figure 5. ResNet-50 Layered Architecture (He K. et al., 2016)
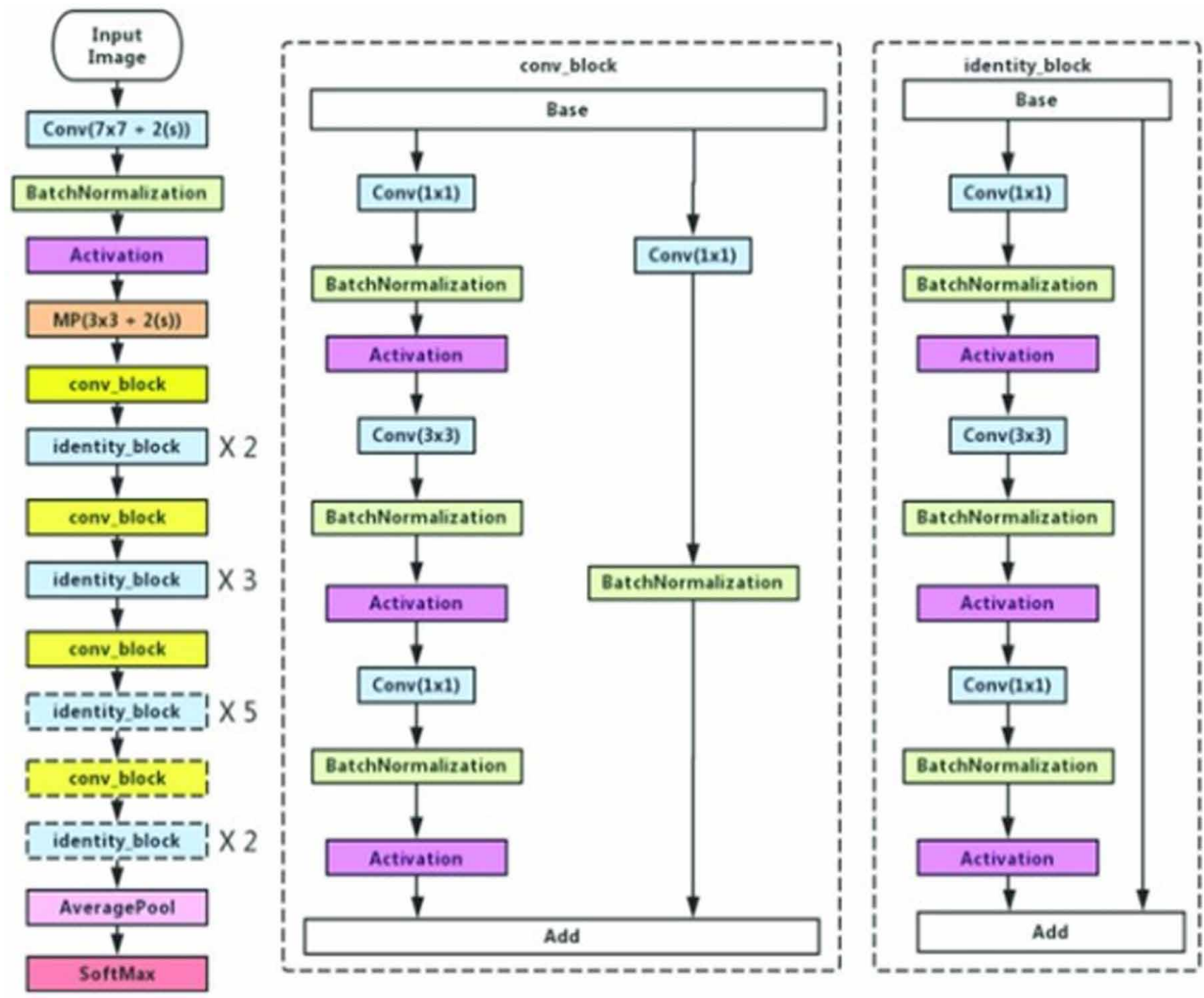

Recall: Recall shows the percentage of relevant results that classify accurately. It is a ratio of true positive and total actual positive.

Recall $=\frac{T P}{T P+F N}$

F1 Score: It is a weighted average of precision and recall. If we have an uneven dataset it is more beneficial.

F1 Score $=2 * \frac{\text { Precision }^{*} \text { Recall }}{\text { Precision }+ \text { Recall }}$

\section{EXPERIMENT RESULT}

To test the proposed model's efficiency, we have implemented all the pre-trained models into the google colab. Google Colab is a free cloud that offers a Jupyter notebook environment that Google 


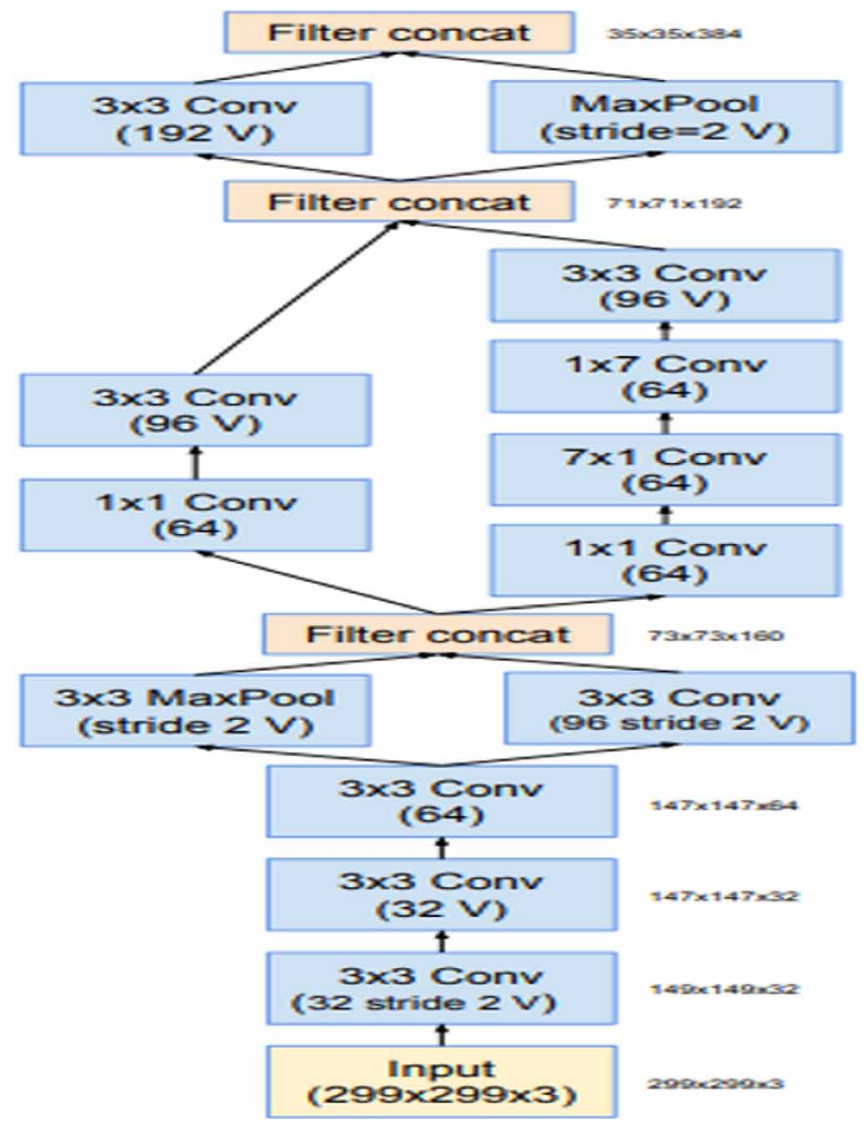

produces for a researcher. It supports both GPU and TPU. The Python programming language is used for simulation purposes. All input images are resized to $224 * 224$ in VGG-19 and ResNet-50, while in Inception ResNetV2 all images are resized to $299 * 299$. To train the proposed model fit_generator method is used. Table 9 depicts the training environment.

a) VGGNet- 19

The new customized VGGNet has 32 layers where the model will be trained only for the ten newly added layers. Figure 7 shows the confusion matrix for training, validation and testing dataset for a proposed model.

The performance of the new model has been summarized in Figure 7 and Tables 10-12. It is observed that during training, the model does not learn any pattern from the input image. The model's accuracy during the training and validation stage is 50\%. It can also be seen in figure 8 and figure 9 which shows the training and validation accuracy and loss respectively.

Here 0 represents Covid class and 1 shows Normal class. It is observed that this model classified all X-Ray images as a Normal class. It shows that VGG-19 model is not a good choice for the Covid detection. Figure 10-12 shows the classification of the X- ray images for training, validation and testing phase. 
International Journal of Healthcare Information Systems and Informatics

Volume $17 \cdot$ Issue 2

Table 7. Comparative Analysis of Total Parameter, Trainable Parameter and Training Time

\begin{tabular}{|l|l|l|l|l|l|}
\hline Model Name & $\begin{array}{c}\text { Total Number } \\
\text { of Layers after } \\
\text { Removing Top } \\
\text { Layers }\end{array}$ & $\begin{array}{c}\text { Total Number } \\
\text { of Layers after } \\
\text { Adding New } \\
\text { Layers }\end{array}$ & Total Parameter & \multicolumn{1}{|c|}{$\begin{array}{c}\text { Trainable } \\
\text { Parameter }\end{array}$} & $\begin{array}{c}\text { Training Time } \\
\text { (second) }\end{array}$ \\
\hline VGGNet-19 & 22 & 32 & $23,699,521$ & 52,48001 & 2158 \\
\hline RestNet-50 & 175 & 185 & $28,835,713$ & 5248001 & 3945 \\
\hline $\begin{array}{l}\text { Inception } \\
\text { ResNetV2 }\end{array}$ & 780 & 790 & $59,060,449$ & 5248001 & 21452 \\
\hline
\end{tabular}

Table 8. Confusion Matrix

\begin{tabular}{|l|l|l|}
\hline & \multicolumn{1}{|c|}{$\begin{array}{c}\text { Predicted (P) } \\
\text { Normal }\end{array}$} & $\begin{array}{c}\text { Predicted (N) } \\
\text { Covid }\end{array}$ \\
\hline $\begin{array}{l}\text { Actual }(\mathrm{P}) \\
\text { Normal }\end{array}$ & TP & FN \\
\hline $\begin{array}{l}\text { Actual }(\mathrm{N}) \\
\text { Covid }\end{array}$ & FP & TN \\
\hline
\end{tabular}

Table 9. Training Setup

\begin{tabular}{|l|l|}
\hline \multicolumn{1}{|c|}{ No. of Epochs } & \multicolumn{1}{c|}{$\mathbf{5 0}$} \\
\hline Batch Size & 32 \\
\hline Learning Rate & 0.01 \\
\hline Multiprocessing & False \\
\hline Shuffle & False \\
\hline Workers & 1 \\
\hline
\end{tabular}

Figure 7. Confusion Matrix for (a) Training Data, (b) Validation Data, (c) Test Data

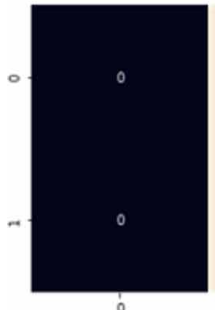

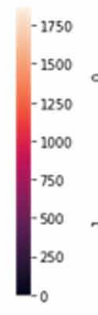

(a)

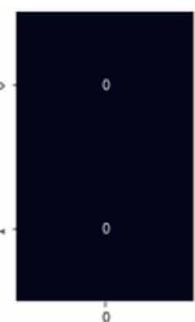

(b)

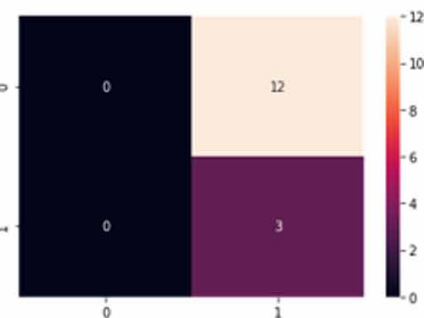

(c) 
Table 10.

\begin{tabular}{|c|c|c|c|c|}
\hline \multicolumn{5}{|c|}{ Table 10: Classification Report for Training Phase } \\
\hline & precision & recall & fl-score & support \\
\hline 0 & 0.00 & 0.00 & 0.00 & 1860 \\
\hline 1 & 0.50 & 1.00 & 0.67 & 1859 \\
\hline Accuracy & & & 0.499 & 3719 \\
\hline macro avg & 0.25 & 0.50 & 0.33 & 3719 \\
\hline weighted avg & 0.25 & 0.50 & 0.33 & 3719 \\
\hline
\end{tabular}

Table 11.

\begin{tabular}{|c|c|c|c|c|}
\hline \multicolumn{5}{|c|}{ Table 11: Classification Report for Validation Phase } \\
\hline & precision & recall & fl-score & support \\
\hline 0 & 0.00 & 0.00 & 0.00 & 200 \\
\hline 1 & 0.50 & 1.00 & 0.67 & 200 \\
\hline Accuracy & & & 0.5 & 400 \\
\hline macro avg & 0.25 & 0.50 & 0.33 & 400 \\
\hline weighted avg & 0.25 & 0.50 & 0.33 & 400 \\
\hline
\end{tabular}

Table 12.

Table 12: Classitication Keport tor lesting Phase
\begin{tabular}{|c|c|c|c|c|}
\hline & precision & recall & fl-score & support \\
\hline 0 & 0.00 & 0.00 & 0.00 & 12 \\
\hline 1 & 0.20 & 1.00 & 0.33 & 3 \\
\hline Accuracy & & & 0.2 & 15 \\
\hline macro avg & 0.10 & 0.50 & 0.17 & 15 \\
\hline weighted avg & 0.04 & 0.20 & 0.07 & 15 \\
\hline
\end{tabular}


International Journal of Healthcare Information Systems and Informatics

Volume $17 \cdot$ Issue 2

Figure 8. Training and Validation Accuracy Progress

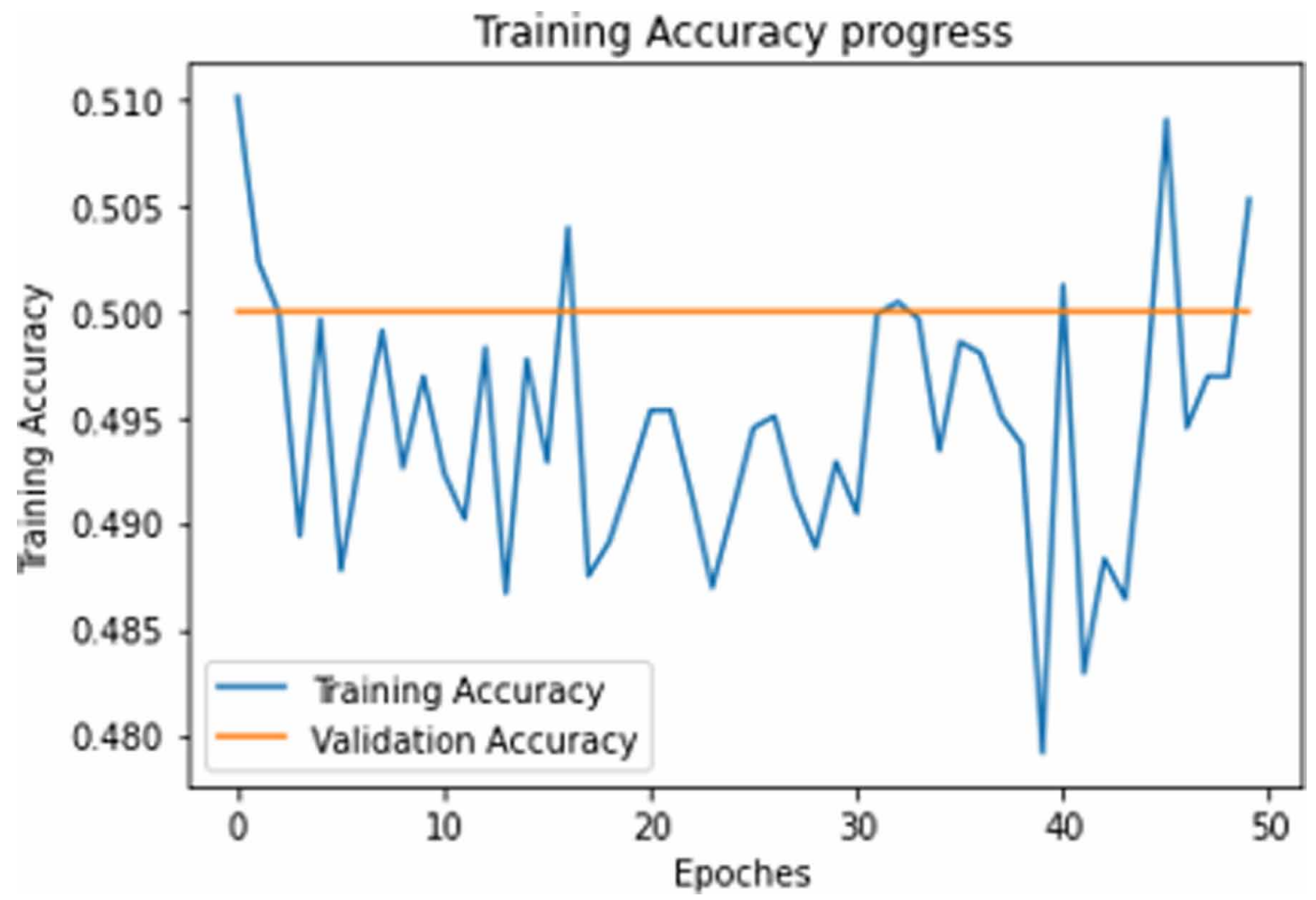

Figure 9. Training and Validation Accuracy Progress

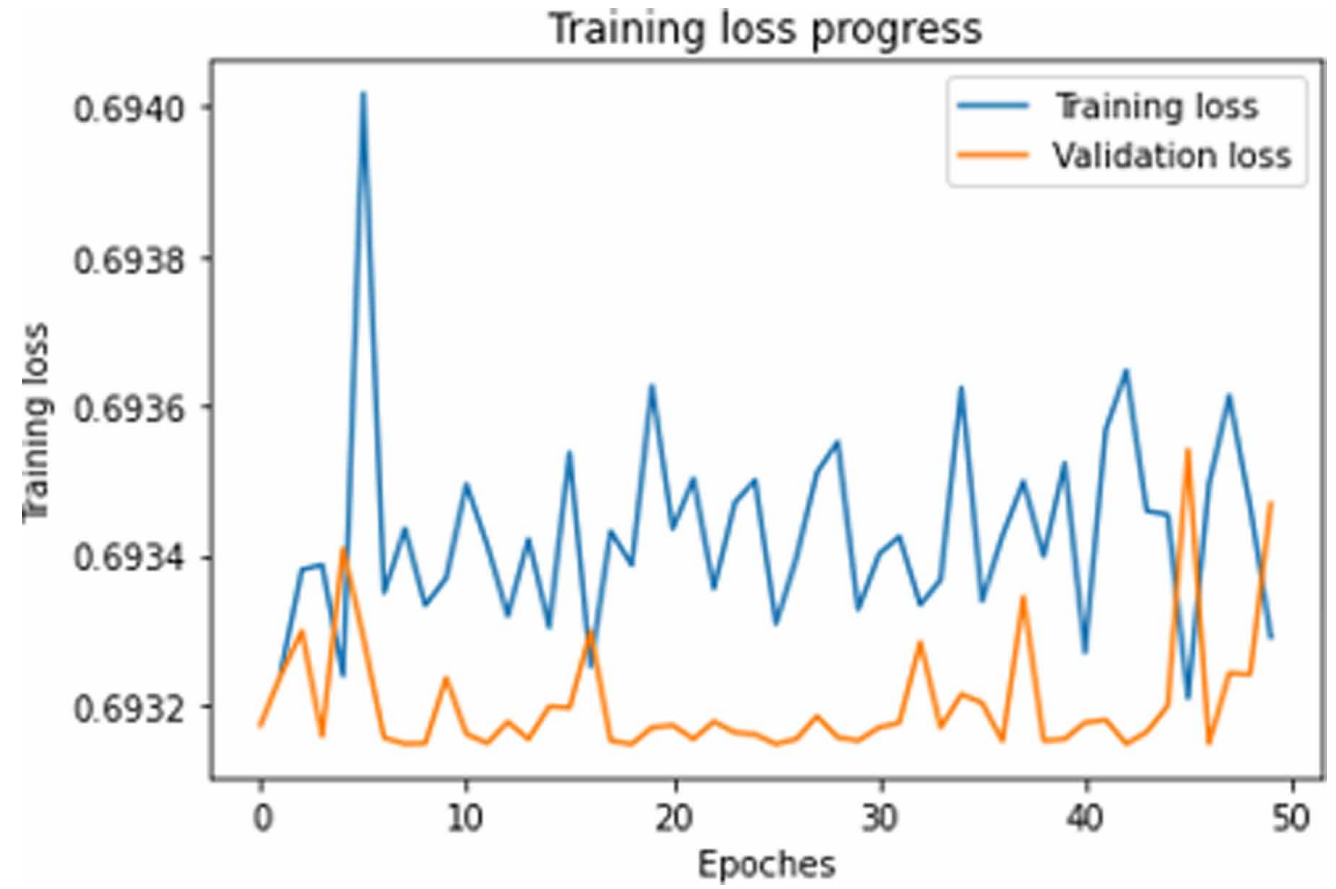


Figure 10. Chest X-Ray Image Classification During Training Phase
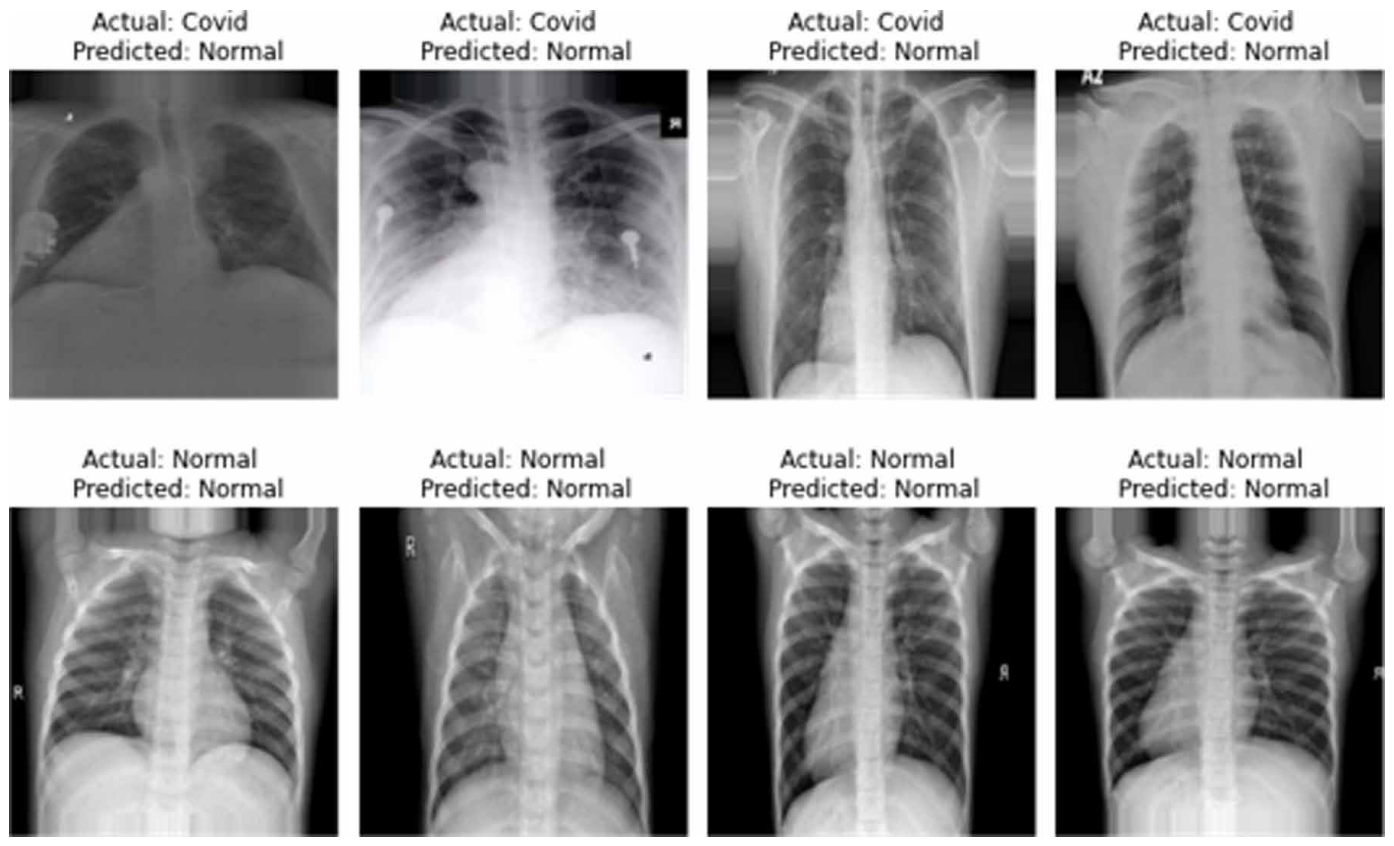

Figure 11. Chest X-Ray Image Classification During Validation Phase

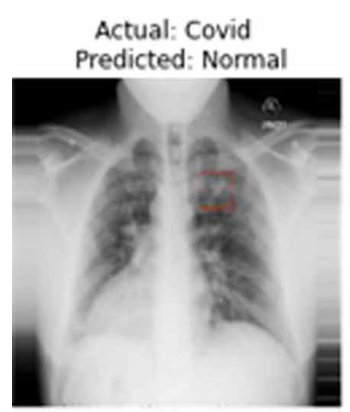

Actual: Normal Predicted: Normal

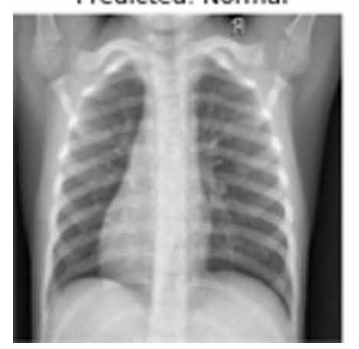

Actual: Covid Predicted: Normal

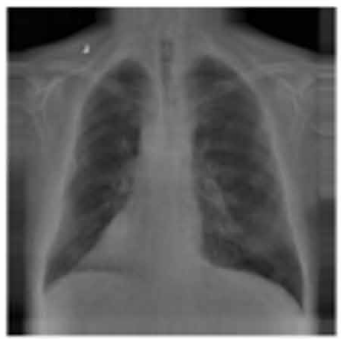

Actual: Normal Predicted: Normal

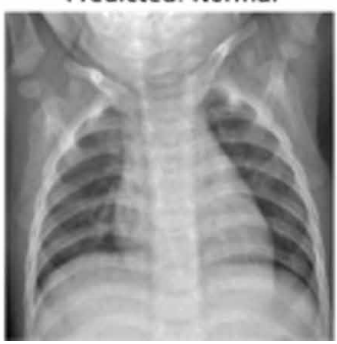

Actual: Covid Predicted: Normal

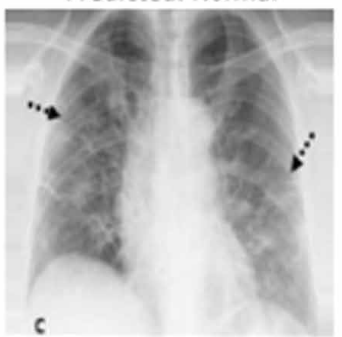

Actual: Normal Predicted: Normal

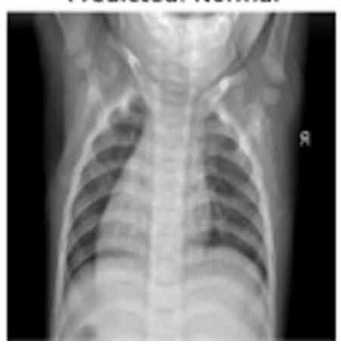

Actual: Covid Predicted: Normal

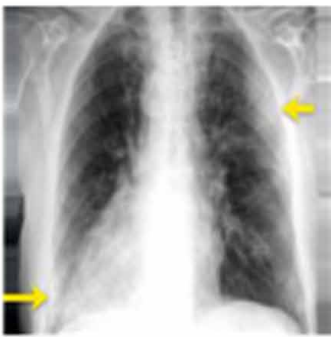

Actual: Normal Predicted: Normal

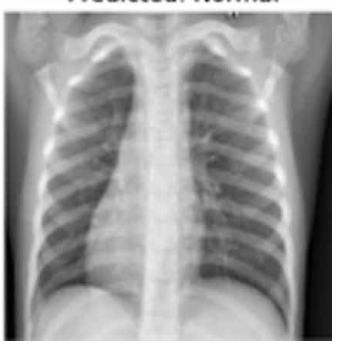


Figure 12. Chest X-Ray Image Classification During Testing Phase

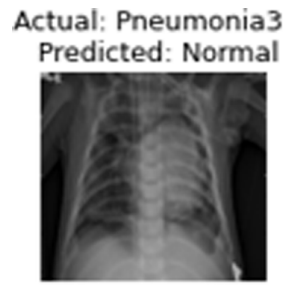

Actual: Normal1 Predicted: Normal

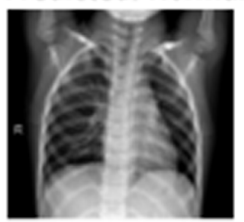

Actual: Covid2 Predicted: Normal

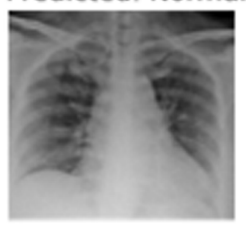

Actual: ARDS2

Predicted: Normal

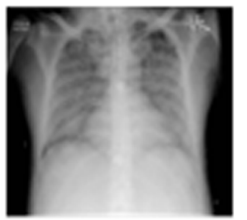

Actual: SARS3 Predicted: Normal

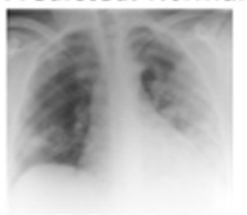

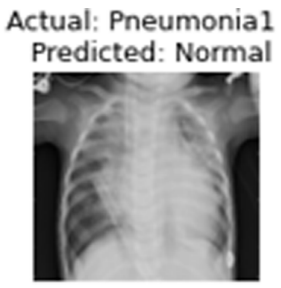

Actual: Normal2 Predicted: Normal

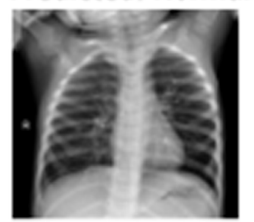

Actual: Covid1 Predicted: Normal

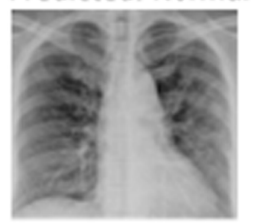

Actual: ARDS1 Predicted: Normal

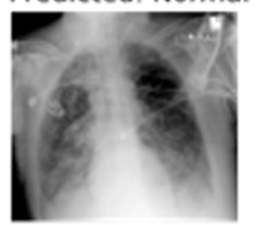

Actual: SARS1 Predicted: Normal

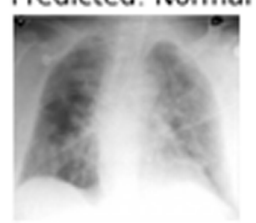

Actual: Pneumonia2 Predicted: Normal

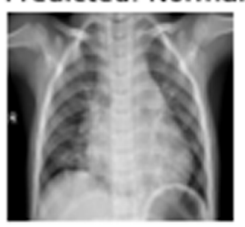

Actual: Normal3 Predicted: Normal

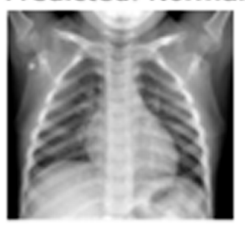

Actual: Covid3 Predicted: Normal

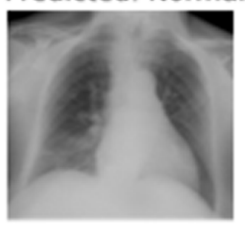

Actual: ARDS3 Predicted: Normal

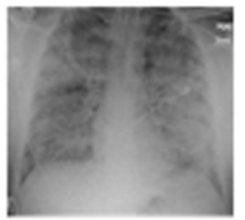

Actual: SARS2 Predicted: Normal

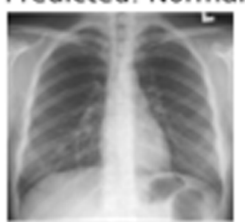

b) ResNet-50

The total number of layers in the new model is 185 . Model is trained only for newly added layers. Figure 13 shows the confusion matrix for the training, validation, and testing phase for the proposed model developed from the ResNet-50 pre-trained model.

Figure 13 and table 13-15 shows the performance of the new model. The training, validation and testing accuracy of the model is $99 \%, 98 \%$ and $87 \%$ respectively. This model can identify Covid, ARDR and SARS disease with higher accuracy, but in some cases, Pneumonia patients are classified as a Normal person. Figure 14-16 shows the X-ray images' classification for the training, validation and testing phase. 
c) InceptionResNetV2

Figure 13. Confusion Matrix for (a) Training Data, (b) Validation Data, (c) Test Data
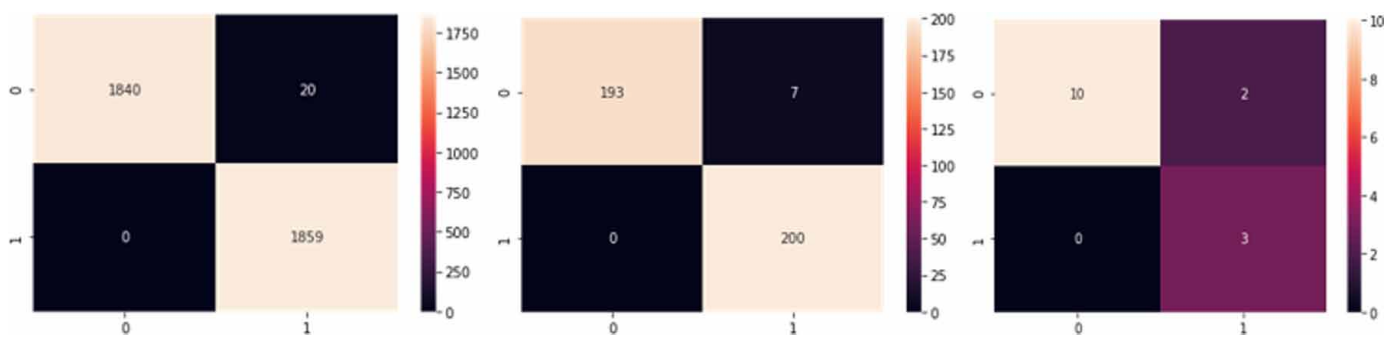

Table 13.

\begin{tabular}{|c|c|c|c|c|}
\hline \multicolumn{5}{|c|}{ Table 13: Classification Report for Training } \\
\hline & precision & recall & fl-score & support \\
\hline 0 & 1.00 & 0.99 & 0.99 & 1860 \\
\hline 1 & 0.99 & 1.00 & 0.99 & 1859 \\
\hline Accuracy & & & 0.99 & 3719 \\
\hline macro avg & 0.99 & 0.99 & 0.99 & 3719 \\
\hline weighted avg & 0.99 & 0.99 & 0.99 & 3719 \\
\hline
\end{tabular}

Table 14.

Table 14: Classification Report for Validation

\begin{tabular}{|c|c|c|c|c|}
\hline & precision & recall & fl-score & support \\
\hline 0 & 1.00 & 0.96 & 0.98 & 200 \\
\hline 1 & 0.97 & 1.00 & 0.98 & 200 \\
\hline Accuracy & & & 0.98 & 400 \\
\hline macro avg & 0.98 & 0.98 & 0.98 & 400 \\
\hline weighted avg & 0.98 & 0.98 & 0.98 & 400 \\
\hline
\end{tabular}

Table 15.

\begin{tabular}{|c|c|c|c|c|}
\hline \multicolumn{5}{|c|}{ Table 15: Classitication Report for lesting } \\
\hline & precision & recall & fl-score & support \\
\hline 0 & 1.00 & 0.83 & 0.91 & 12 \\
\hline 1 & 0.60 & 1.00 & 0.75 & 15 \\
\hline Accuracy & 0.80 & 0.92 & 0.87 & 15 \\
\hline macro avg & 0.92 & 0.87 & 0.93 & 15 \\
\hline weighted avg & & & 0.88 & \\
\hline
\end{tabular}


Figure 14. Chest X-Ray Image Classification During Training Phase
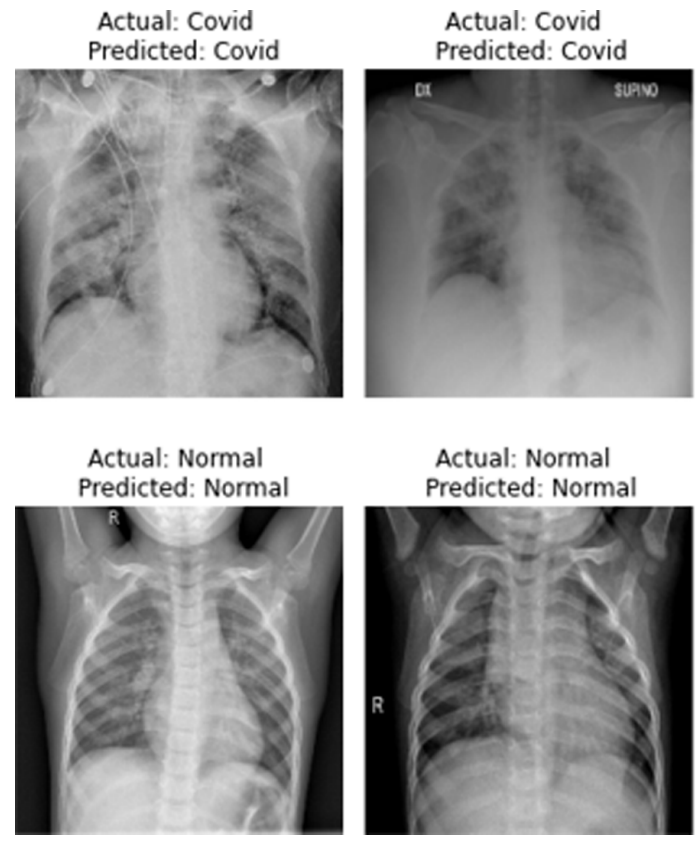

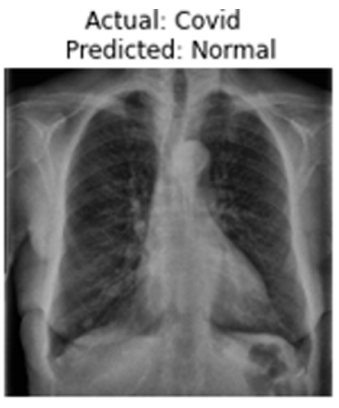

Actual: Normal Predicted: Normal

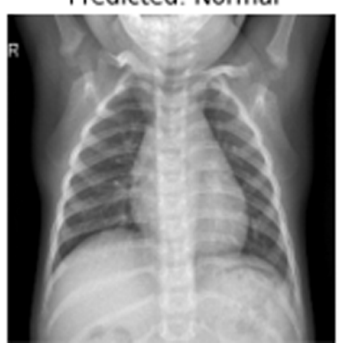

Actual: Covid Predicted: Covid

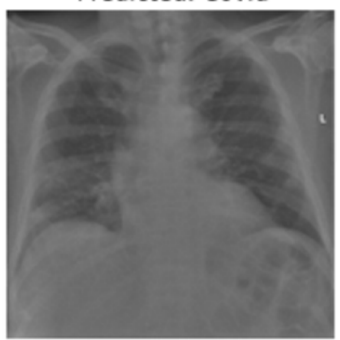

Actual: Normal Predicted: Normal

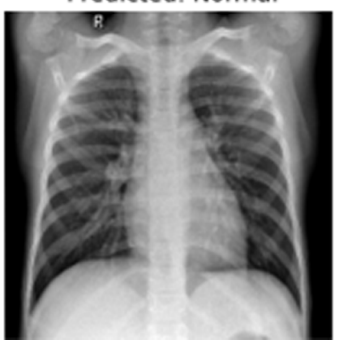

Figure 15. Chest X-Ray Image Classification During Validation Phase

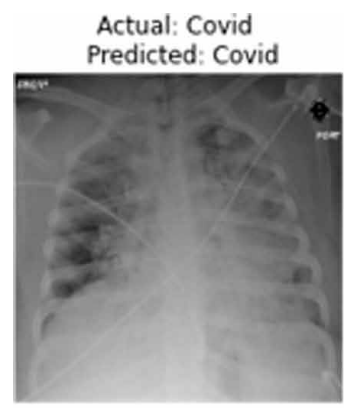

Actual: Normal Predicted: Normal

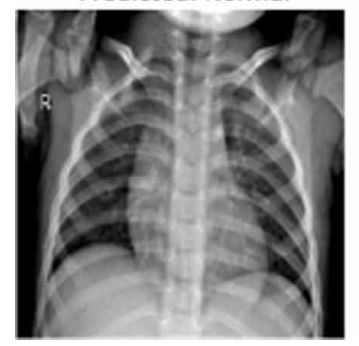

Actual: Covid

Predicted: Covid

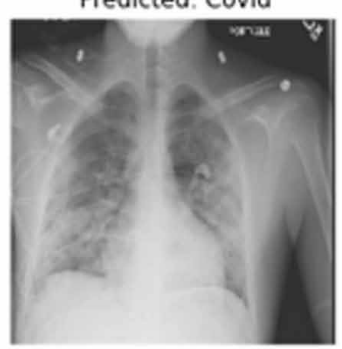

Actual: Normal Predicted: Normal

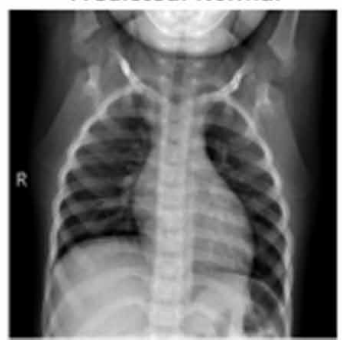

Actual: Covid

Predicted: Covid

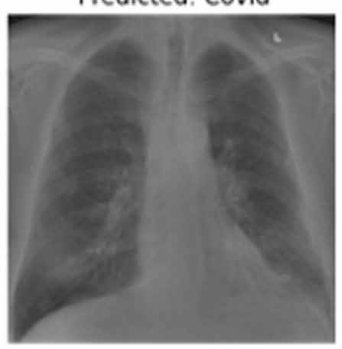

Actual: Normal Predicted: Normal

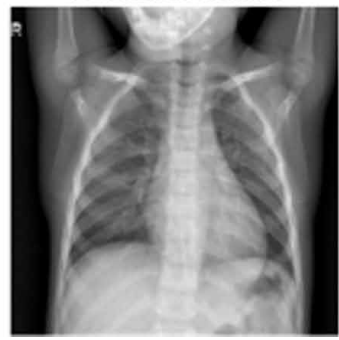

Actual: Covid

Predicted: Normal

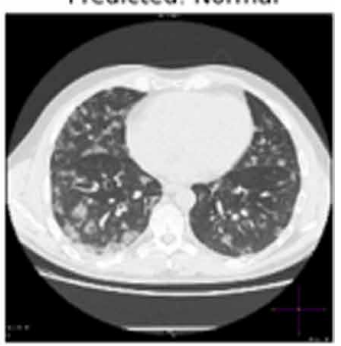

Actual: Normal Predicted: Normal

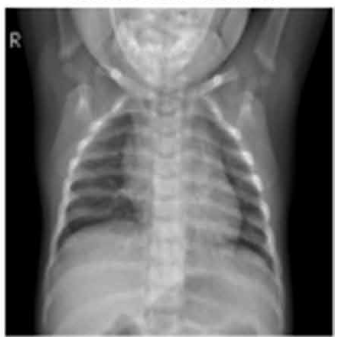


Figure 16. Chest X-Ray Image Classification During Testing Phase

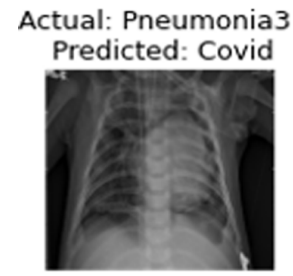

Actual: Normal1 Predicted: Normal

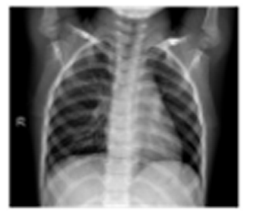

Actual: Covid2 Predicted: Covid

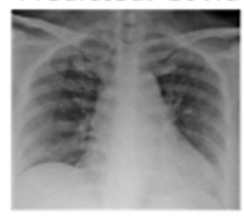

Actual: ARDS2

Predicted: Covid

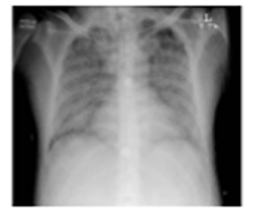

Actual: SARS3

Predicted: Covid

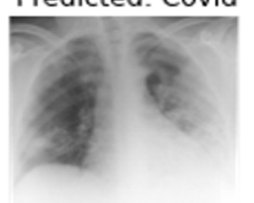

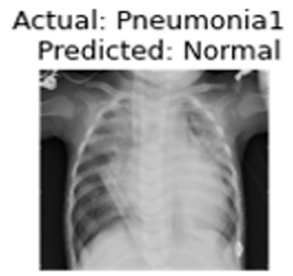

Actual: Normal2 Predicted: Normal

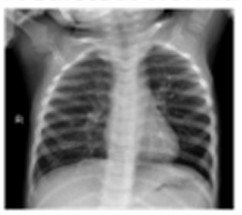

Actual: Covid1 Predicted: Covid

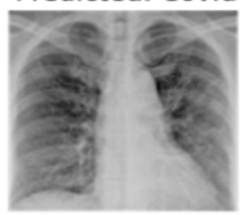

Actual: ARDS1 Predicted: Covid

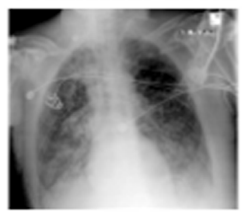

Actual: SARS1 Predicted: Covid

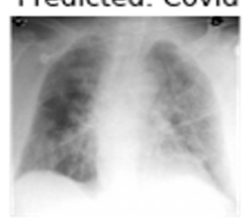

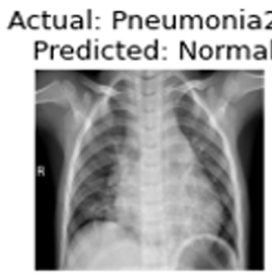

Actual: Normal3 Predicted: Norma

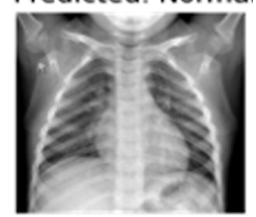

Actual: Covid3 Predicted: Covid

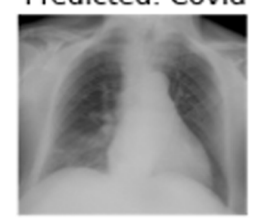

Actual: ARDS3 Predicted: Covid

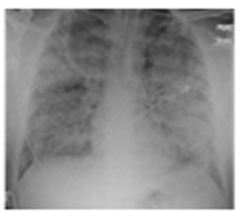

Actual: SARS2

Predicted: Covid

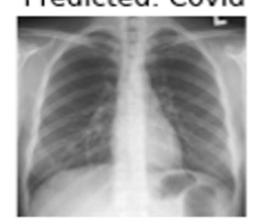

The new InceptionResNetV2 model has 790 layers. Model is train only for newly added layers. Figure 17 shows the confusion matrix for the training, validation and testing dataset for the proposed model developed from InceptionResNetV2 pre-trained model.

The model's performance is shown in figure 17 and table 16-18. The training, validation and testing accuracy of the model is $99.2 \%, 98 \%$ and $94 \%$ respectively. This model is able to classify more reliably Covid, ARDR and SARS, but in some cases, Pneumonia patients are classified as a Normal class. After analyzing the confusion matrix and classification report of the testing phase, it is observed that the InceptionNet model classified a smaller number of Pneumonia images as Normal as compare to the VGG-19 model. As our main objective is to predict whether the person is healthy (Normal) or not so this model is good as compare to the ResNet model. Figure 18-20 shows the classification of the X-ray images for training, validation and testing phase.

After comparing the table from 10-18 and figure from 7-20, it can be illustrated Inception Resnet_v2 is a better choice for the Covid classification compared to the VGG-19 because it gives 
Figure 17. Confusion Matrix for (a) Training Data, (b) Validation Data, (c) Test Data
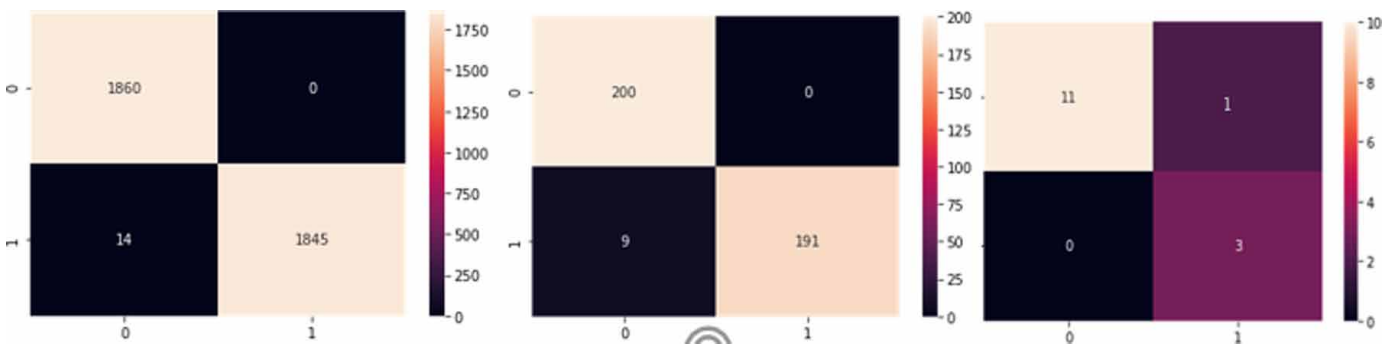

Table 16.

Table 16: Classification Report for Training

\begin{tabular}{|c|c|c|c|c|}
\hline & precision & recall & fl-score & support \\
\hline 0 & 1.00 & 0.93 & 0.96 & 1860 \\
\hline 1 & 0.94 & 1.00 & 0.97 & 1859 \\
\hline Accuracy & & & 0.99 & 3719 \\
\hline macro avg & 0.97 & 0.97 & 0.97 & 3719 \\
\hline weighted avg & 0.97 & 0.97 & 0.97 & 3719 \\
\hline
\end{tabular}

Table 17.

\begin{tabular}{|c|c|c|c|c|}
\hline \multicolumn{5}{|c|}{ Table 17: Classification Report for Validation } \\
\hline & precision & recall & fl-score & support \\
\hline 0 & 0.96 & 1.00 & 0.98 & 200 \\
\hline 1 & 1.00 & 0.95 & 0.98 & 200 \\
\hline Accuracy & & & 0.98 & 400 \\
\hline macro avg & 0.98 & 0.98 & 0.98 & 400 \\
\hline weighted avg & 0.98 & 0.98 & 0.98 & 400 \\
\hline
\end{tabular}

Table 18.

\begin{tabular}{|c|c|c|c|c|}
\hline \multicolumn{5}{|c|}{ Table 18: Classitication Keport tor lesting } \\
\hline & precision & recall & fl-score & support \\
\hline 0 & 1.00 & 0.92 & 0.96 & 12 \\
\hline 1 & 0.75 & 1.00 & 0.86 & 15 \\
\hline Accuracy & & & 0.94 & 15 \\
\hline macro avg & 0.91 & 0.93 & 0.93 & 15 \\
\hline weighted avg & 0.95 & 0.91 & 0.93 & \\
\hline
\end{tabular}


Figure 18. Chest X-Ray Image Classification During Training Phase

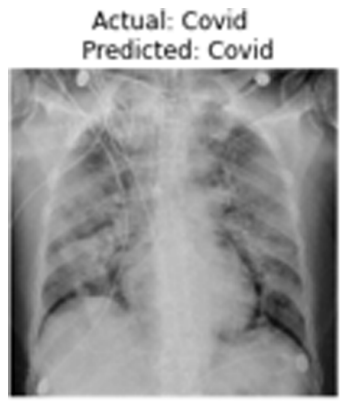

Actual: Normal Predicted: Normal

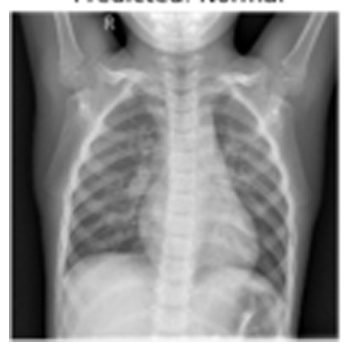

Actual: Covid

Predicted: Covid

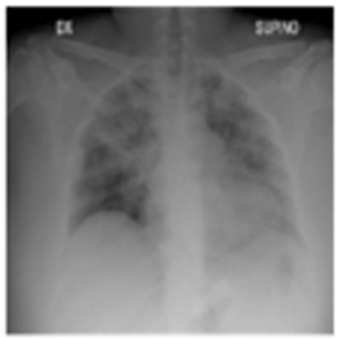

Actual: Normal Predicted: Normal

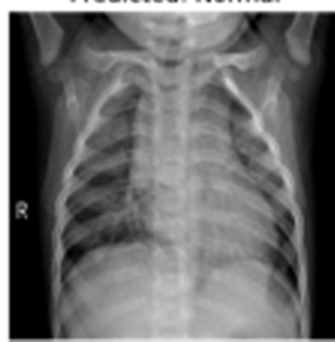

Actual: Covid Predicted: Normal

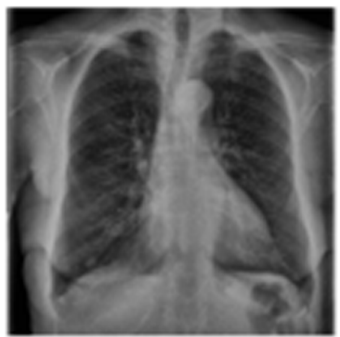

Actual: Normal Predicted: Normal

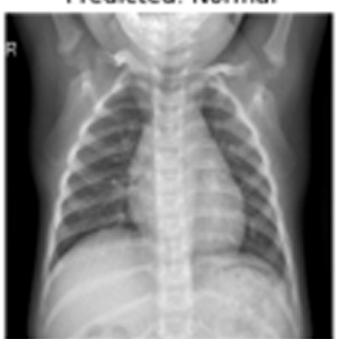

Actual: Covid

Predicted: Covid

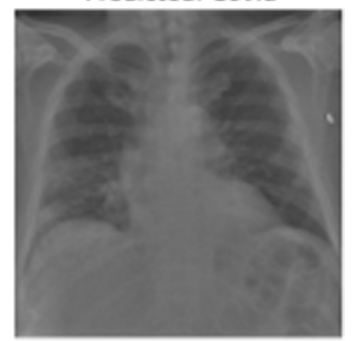

Actual: Normal Predicted: Normal

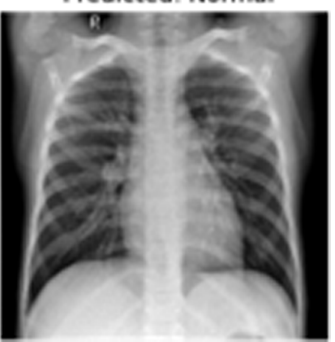

Figure 19. Chest X-Ray Image Classification During Validation Phase

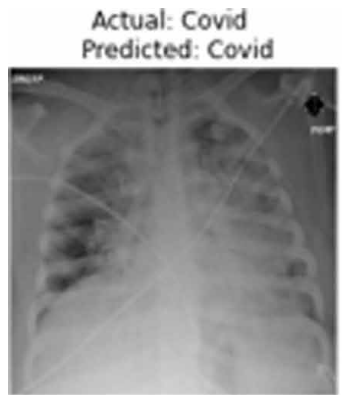

Actual: Normal Predicted: Normal

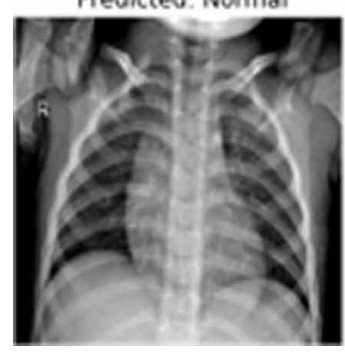

Actual: Covid

Predicted: Covid

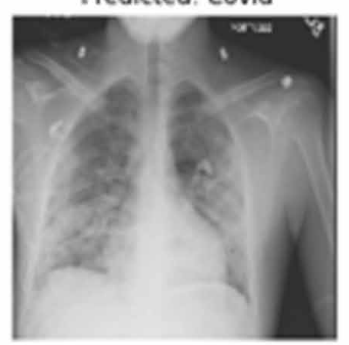

Actual: Normal Predicted: Normal

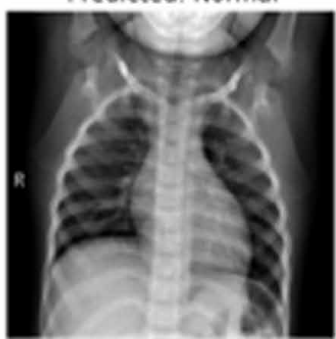

Actual: Covid

Predicted: Covid

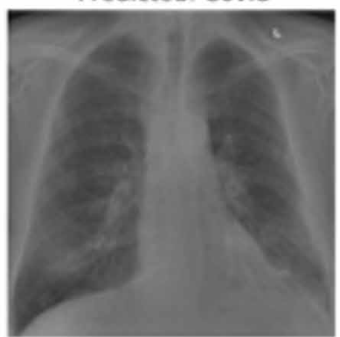

Actual: Normal Predicted: Normal

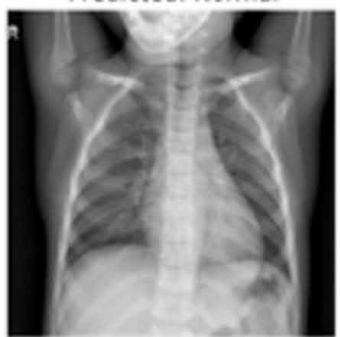

Actual: Covid Predicted: Normal

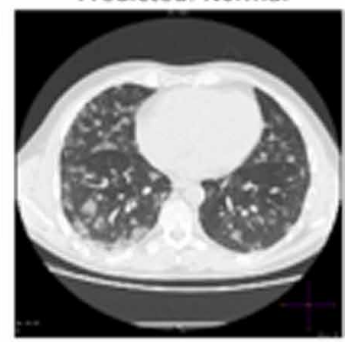

Actual: Nomal Predicted: Normal

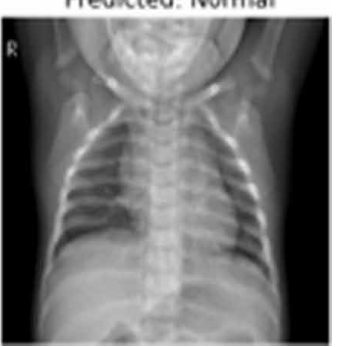


Figure 20. Chest X-Ray Image Classification During Testing Phase

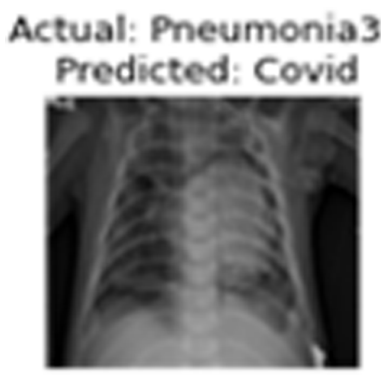

Actual: Normal1

Predicted: Normal

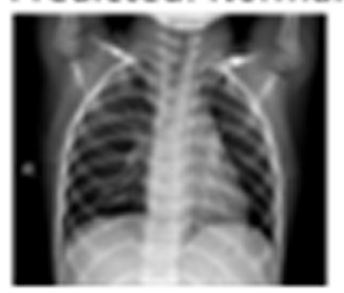

\section{Actual: Covid2} Predicted: Covid

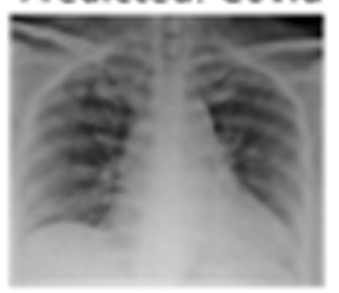

Actual: ARDS2 Predicted: Covid

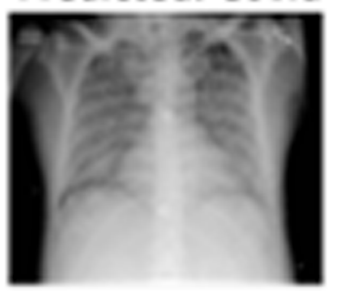

Actual: SARS 3 Predicted: Covid

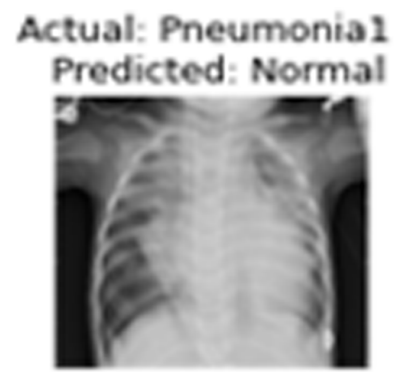

\section{Actual: Normal2}

Predicted: Normal

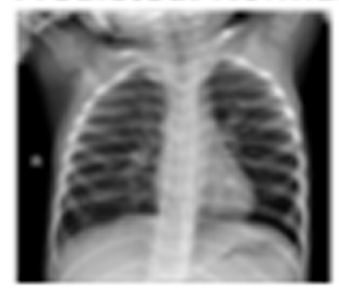

Actual: Covid 1

Predicted: Covid

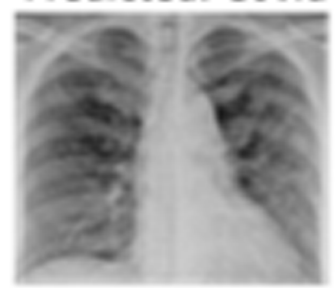

Actual: ARDS1 Predicted: Covid

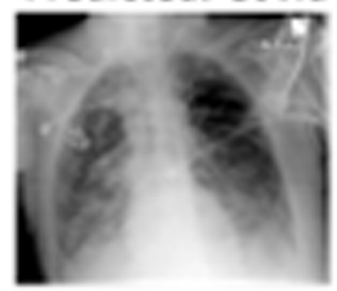

Actual: SARS1 Predicted: Covid

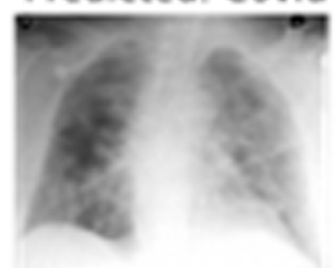

Actual: Pneumonia2

Predicted: Corvid

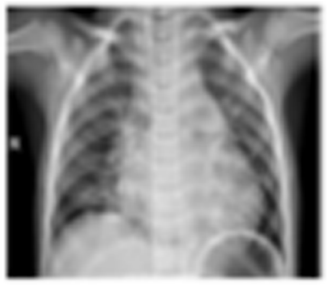

\section{Actual: Normal3}

Predicted: Normal

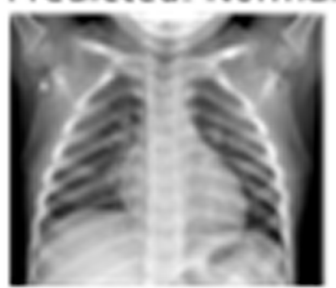

Actual: Covid 3

Predicted: Covid

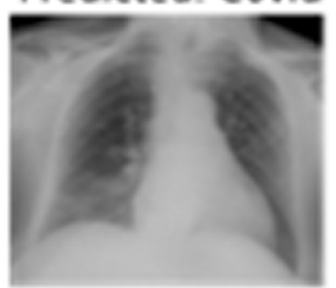

Actual: ARDS3

Predicted: Covid

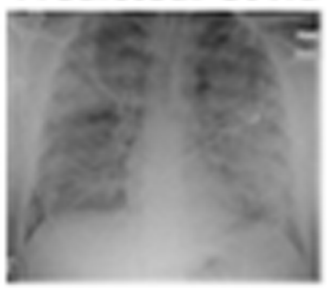

Actual: SARS2

Predicted: Covid

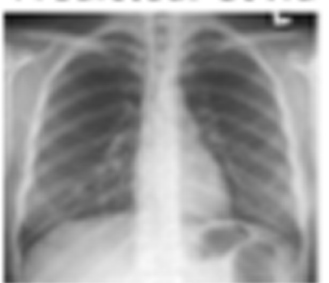


less number of false-positive rate. Figure 21 depicts the comparative analysis of training, validation and testing accuracy of VGGNet, ResNet and Inception ResNet.

Figure 21. Comparative Analysis of Training, Validation and Testing Accuracy of VGGNet, ResNet and Inception ResNet

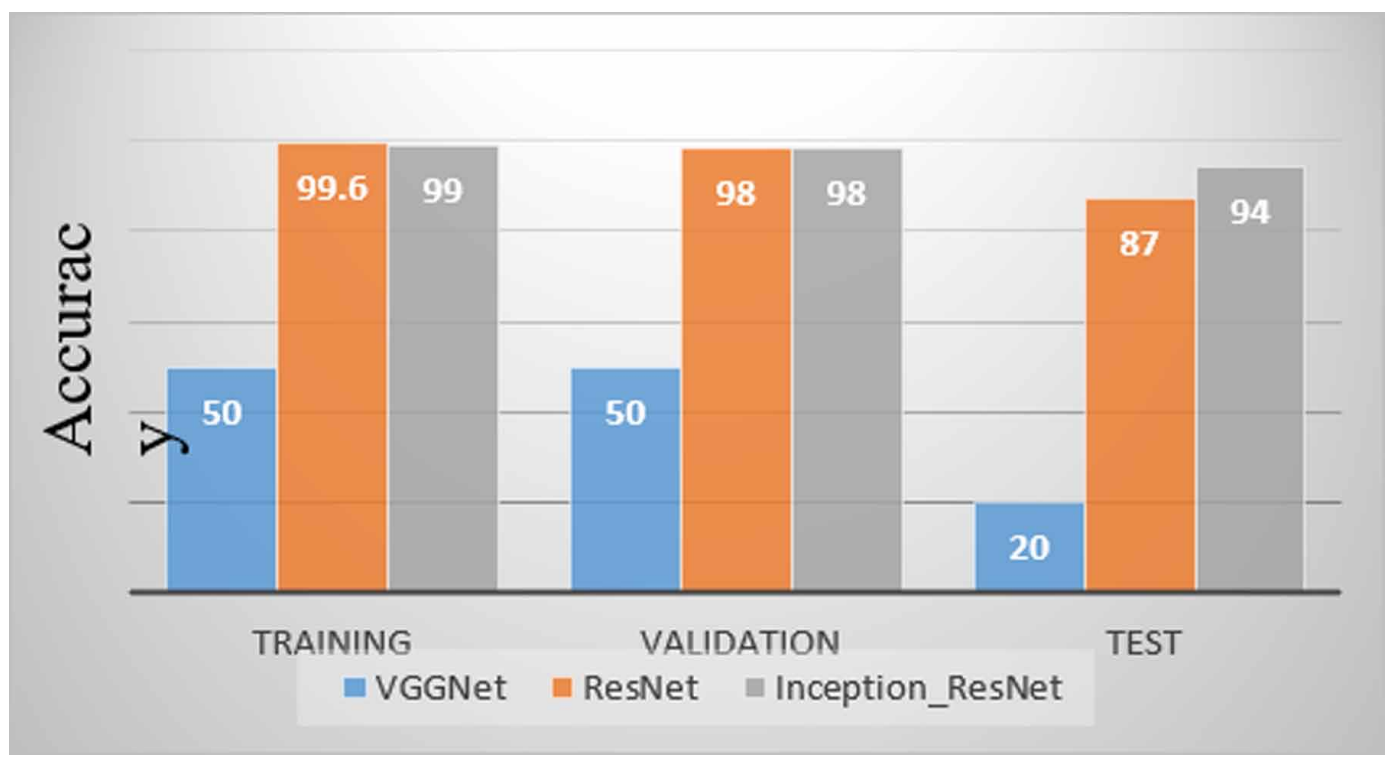

\section{CONCLUSION}

Due to the exponential growth of Covid-19 patients, many countries phase the problem of inadequate clinical diagnostic services like limited test kits, ventilators etc. This paper proposed a Deep Learning based automatic Covid detection system from chest X-Ray images. X-Ray test is cheapest and easily available resources that can help to detect Covid patient in the early stage. Due to the less number of Covid images, we used the pre-trained model and customized it for Covid detection. This paper shows the comparative analysis of three pre-trained model VGGNet-19, ResNet and Inception_ResNet. To minimize the possibility of the misclassification where Covid patient classifies as Normal, we have not trained our model for the Pneumonia, ARDS and SARS so our model classified all these three diseases as Covid. The reason for this is to minimize the false-positive rate (i.e., Covid patient classified as Normal). Experiment results shows that Inception Resnet_v2 gives more accuracy as compare to VGGNet and ResNet.

\section{DISCLOSURE STATEMENT}

We declare that we have no conflicts of interest to disclose.

\section{FUNDING}

The authors have not received research grants from any company. 


\section{REFERENCES}

Abbas, A., Abdelsamea, M. M., \& Gaber, M. M. (2020). Classification of COVID-19 in Chest X-Ray Images using DeTraC Deep Convolutional Neural Network. Applied Intelligence, 1-11.

Asif, S., Wenhui, Y., Join, H., Tao, Y., \& Jinhai, S. (2020). Classification of COVID-19 from Chest X-ray images using Deep Convolutional Neural Networks. MedReiv. 1-14.

Asnaoui, K. E., \& Chawki, Y. (2020). Using X-Ray Images and Deep Learning for Automated Detection of Coronavirus Disease. Journal of Biomolecular Structure \& Dynamics, 1-13.

Bhandary, A., Prabhu, G. A., Rajinikanth, V., Thanaraj, K. P., Satapathy, S. C., \& Robbins, D. E. (2020). DeepLearning Framework to Detect Lung Abnormality-A Study with Chest X-Ray and Lung CT Scan Images. Pattern Recognition Letters, 129, 271-278.

Cohen. (2020). Covid Chest X-ray Dataset. Available at https://github.com/ieee8023/covid-chestxray-dataset

Farooq, M., \& Hafeez, A. (2020). COVID-ResNet: A Deep Learning Framework for Screening of COVID19. Radiographs, 1-6.

Gozes, O., Adar, M. F., Greenspan, H., Browning, P. D., Zhang, H., Ji, W., Bernheim, A., \& Siegel, E. (2020). Rapid AI development cycle for the Coronavirus (COVID-19) Pandemic: Initial Results for Automated Detection \& Patient Monitoring Using Deep Learning CT Image Analysis. Radiology, 1-22.

He, K., Zhang, X., Ren, S., \& Sun, J. (2016). Deep Residual Learning for Image Recognition. Proceeding of IEEE Conference on Computer Vision and Pattern Recognition (CVPR), 1-12.

Hemdan, E. D., Shouman, M. A., \& Karar, M. E. (2020). COVIDX-Net: A Framework of Deep Learning Classifiers to Diagnose COVID-19 in X-Ray Images. Electrical Engineering and Systems Science, 1-14.

Iiyas, M., Rehman, H., \& Ali, A. N. (2020). Detection of Covid-19 From Chest X-ray Images Using Artificial Intelligence: An Early Review. Electrical Engineering and Systems Science, 1-8.

Ji, Q., Huang, J., He, W., \& Sun, Y. (2019). Optimized Deep Convolutional Neural Networks for Identification of Macular Diseases from Optical Coherence Tomography Images. Algorithm., 12(3), 1-12.

Kermany, . (2018). Chest $x$-ray \& CT dataset. Available at https://www.kaggle.com/paultimothymooney/chestxray-pneumonia

Khan, A. I., Shah, J. L., \& Bhat, M. M. (2020). CoroNet: A Deep Neural Network for Detection and Diagnosis of Covid-19 from Chest X-ray Images. Computer Methods and Programs in Biomedicine, 196, 1-9. doi:10.1016/j. cmpb.2020.105581 PMID:32534344

Khan, S. H., Sohail, A., Zafar, M. M., \& Khan, A. (2020). Coronavirus Disease Analysis using Chest X-ray Images and a Novel Deep Convolutional Neural Network. Academic Press.

Lin, M., Chen, Q., \& Yan, S. (2014). Network in Network. Neural and Evolutionary Computing, 1-10.

Makris, A., Kontopoulos, L., \& Tserpes, K. (2020). COVID-19 Detection from Chest X-Ray Images using Deep Learning and Convolutional Neural Networks. MedReiv. 1-14.

Narin A., Kaya C. \& Pamuk Z. (2020). Automatic Detection of Coronavirus Disease (COVID-19) Using X-Ray Images and Deep Convolutional Neural Networks. Electrical Engineering and Systems Science, 1-31.

Ozturk, T., Talo, M., Yildirim, E. A., Baloglu, U. B., Yildirim, O., \& Acharya, U. R. (2020). Automated Detection of COVID-19 Cases Using Deep Neural Networks with X-ray Images. Computers in Biology and Medicine, 121, 1-11. doi:10.1016/j.compbiomed.2020.103792 PMID:32568675

Rajpurkar, P., Irvin, J., Zhu, K., Yang, B., Mehta, H., Duan, T., Ding, D., Bagul, A., Ball, R. L., Langlotz, C., Shpanskaya, K., Lungren, M. P., \& Ng, A. Y. (2017). Chexnet: Radiologist-Level Pneumonia Detection on Chest $X$-Rays With Deep Learning. Academic Press.

Shan, F., Gao, Y., Wang, J., Shi, W., Shi, N., Han, M., Xue, Z., Shen, D., \& Shi, Y. (2020). Lung Infection Quantification of COVID-19 in CT Images With Deep Learning, Computer Vision and Pattern Recognition. Academic Press. 
Simonyan, K., \& Zisserman, A. (2015). Very Deep Convolutional Networks for Large-Scale Image Recognition. Proceeding of the international Conference on Learning Representations (ICLR), 1-14.

Szegedy, C., Loffe, S., Vanhoucke, V., \& Alemi, A. A. (2017). Inception-v4, inception-ResNet and the Impact of Residual Connections on Learning. Proceedings of the Thirty-First AAAI Conference on Artificial Intelligence, $4278-4284$.

Wang, W., \& Xia, Y. (2018). Chestnet: A Deep Neural Network for Classification of Thoracic Diseases on Chest Radiography. Computer Vision and Pattern Recognition, 1-8.

Zhang, J., Xie, Y., Li, Y., \& Xia, Y. (2020). COVID-19 Screening on Chest X-Ray Images Using Deep Learning Based Anomaly Detection. Academic Press.

Zu, Z. Y., Jiang, M. D., Xu, P. P., Chen, W., Ni, Q. Q., Lu, G. M., \& Zhang, L. J. (2020). Coronavirus Disease (COVID-19): A Perspective from China. Radiology, 295(3), 1-29. PMID:32083985 